\title{
CUPRATES, MANGANITES, AND COBALTITES: MULTIELECTRON APPROACH TO THE BAND STRUCTURE
}

\author{
M.M. KORSHUNOV ${ }^{1,2}$, S.G. OVCHINNIKOV ${ }^{1,2}$, E.I. SHNEYDER ${ }^{1,3}$, V.A. \\ GAVRICHKOV $^{1,2}$, Yu.S. ORLOV ${ }^{1,2}$, I.A. NEKRASOV ${ }^{4}$, Z.V. PCHELKINA ${ }^{5}$ \\ ${ }^{1}$ L.V. Kirensky Institute of Physics, Siberian Branch of Russian Academy of Sciences, \\ Krasnoyarsk 660036, Russia \\ ${ }^{2}$ Siberian Federal University, Svobodny Prospect 79, Krasnoyarsk 660041, Russia \\ 3 Reshetnev Siberian State Aerospace University, Krasnoyarsk 660014, Russia \\ ${ }^{4}$ Institute for Electrophysics, Russian Academy of Sciences, Ekaterinburg 620016, Russia \\ ${ }^{5}$ Institute for Metal Physics, Russian Academy of Sciences, Ekaterinburg 620219, Russia \\ sgo@iph.krasn.ru
}

Received (Day Month Year)

Revised (Day Month Year)

\begin{abstract}
High- $T_{c}$ superconductors with $\mathrm{CuO}_{2}$ layers, manganites $\mathrm{La}_{1-x} \mathrm{Sr}_{x} \mathrm{MnO}_{3}$, and cobaltites $\mathrm{LaCoO}_{3}$ present several mysteries in their physical properties. Most of them are believed to come from the strongly-correlated nature of these materials. From the theoretical viewpoint, there are many hidden rocks in making the consistent description of the band structure and low-energy physics starting from the Fermi-liquid approach. Here we discuss the alternative method - multielectron approach to the electronic structure calculations for the Mott insulators - called LDA+GTB (local density approximation + generalized tight-binding) method. Its origin is a straightforward generalization of the Hubbard perturbation theory in the atomic limit and the multiband $p-d$ Hamiltonian with the parameters calculated within LDA. We briefly discuss the method and focus on its applications to cuprates, manganites, and cobaltites.
\end{abstract}

Keywords: LDA+GTB method; Strongly-correlated systems; Band structure.

\section{Introduction}

Fermi-liquid approach is one of the most attractive and useful in the modern condensed matter physics. Its cornerstone is a quasiparticle defined as a renormalized electron - the one surrounded by the cloud of excitations. Such concept allows to use a number of the well-developed theoretical tools like diagram technique, path integrals, etc. The strong point of the Fermi-liquid approach is generality but in many cases it becomes a weak point. When it comes to real material-specific questions, the starting point for the study should depend on the particular material's characteristics. Huge leap in this direction was made in mid 60th by Hohenberg, Kohn, and Sham $\frac{1 / 2}{2}$ who formulated a density functional theory (DFT). Because its starting point is the Shrödinger equation for the particular atomic arrangement, orbital, and spin configurations, this theory is often referred to as the 'first principles' or the 
' $a b$ initio' calculations. Augmented with the local density approximation (LDA) or the generalized gradient approximation (GGA) for the initially unknown quantity, exchange-correlation energy, DFT provides quantitative description of the ground state energy and the band structure of various atoms, molecules, and crystalline solids, especially containing $s$ and $p$ atoms (see e.g. ${ }^{3}$ ).

There are many cases, however, when the basic concept of the Fermi-liquid brakes and the system becomes a non-Fermi-liquid. Examples include temperaturedependent metal-insulator transitions in cobaltites and a pseudogap state in cuprates. In this case, one-electron approaches like LDA and GGA give qualitatively wrong results. In particular, LDA failed to describe transition metal oxides with partially filled $3 d$-orbitals. The most pronounced failure is that LDA predicts $\mathrm{La}_{2} \mathrm{CuO}_{4}$ to be a metal whereas experimentally it is an insulator. The root of the problem is the unscreened on-site Coulomb interaction (Hubbard repulsion) ${ }^{4}$. In a single-band system on the mean-field level if Hubbard repulsion $U$ is larger than the bandwidth $W$, it splits the band into two Hubbard subbands with a gap $\propto U$. Spectral weight of a quasiparticle is redistributed between these subbands. At a half-filling, the Fermi level is inside the gap and the system is an insulator. In a multiorbital system, along with the Hubbard repulsion other local interactions like the Hund's exchange $J_{H}$ and the interorbital Hubbard repulsion $U^{\prime}$ are present and provide a rich set of physical properties. Opening of the Hubbard gap and moreover the major role played by the local interactions near the half-filling are beyond the scope of LDA and GGA.

There are several extensions to LDA which includes or simulates the effects of the on-site interactions. One of them is LDA $+\sqrt{5}$ and another one is SIC-LSDA (self-interaction-corrected local spin density approximation) $\sqrt{6}$. Both methods consider local interactions in the Hartree-Fock sense and result in the antiferromagnetic insulator as the ground state for $\mathrm{La}_{2} \mathrm{CuO}_{4}$ contrary to LDA, but the origin of the insulating gap is incorrect. In both LDA $+\mathrm{U}$ and SIC-LSDA, it is formed by the local single-electron states splitted by the spin or orbital polarization. Therefore, the paramagnetic phase above the Néel temperature $T_{N}$ of the undoped $\mathrm{La}_{2} \mathrm{CuO}_{4}$ will be metallic in spite of strong correlation regime $U \gg W$. There is one more significant drawback in these approximations, namely, they disregard the redistribution of the spectral weight between the Hubbard subbands. Latter effect is incorporated in a different approach to ab initio calculations for strongly correlated systems LDA+DMFT (LDA+dynamical mean field theory) ${ }^{7 / 8|9| 10}$. The method is based on the self-consistent procedure where the LDA band structure is used to calculate the electron self-energy in DMFT. DMFT utilizes the fact that in the infinite dimensional limit of the Hubbard model, $D \rightarrow \infty$, the self-energy is momentum independent, $\Sigma(\mathbf{k}, \omega) \rightarrow \Sigma(\omega)$ 11|12|13. The remaining frequency dependence is exact in $D \rightarrow \infty$ limit and carries very important information about dynamical correlations and Mott-Hubbard transition. On the other hand, the spatial correlations become crucial in low-dimensional systems like layered high- $T_{c}$ cuprates. That is 
why the correct band dispersion and spectral intensities for these systems cannot be obtained within LDA+DMFT. Natural extension of this method, LDA+cluster or cellular DMFT 14|15/16/17, and SDFT (spectral density functional theory) 18 provides momentum dependent self-energy and thus allow for the non-local correlations.

Here we are going to discuss the alternative approach - the LDA+GTB method - to study the Mott-Hubbard insulators. From the very beginning the GTB (generalized tight binding) method has been suggested to extend the microscopic band structure calculations to take the strong electronic correlations (SEC) into account in the Mott-Hubbard insulators like the transition metal oxides 19 . Similar to conventional tight binding (TB) approach we start with a particular local electron states (with all multiorbital effects, symmetry and chemistry) and then by a Fourier transform move to the momentum space and obtain a band structure. Because of SEC we can not use free electron local states, our local fermion in a $d$-orbital system is a quasiparticle given by the excitations between multielectron $d^{n}$ and $d^{n \pm 1}$ terms contrary to the conventional TB. In other words, GTB is the strongly correlated version of the TB method. The first computer codes and successful application of GTB has been developed for cuprates 20 . That version used the multiband $p-d$ model for $\mathrm{La}_{2} \mathrm{CuO}_{4} 21$ with a lot of empirical parameters in the Hamiltonian. To "cook" the $a b$ initio approach, the hybrid LDA+GTB method has been developed 22 . Afterward, similar ideas have been used to study the GTB band structure of manganites $\mathrm{La}_{1-x} \mathrm{Sr}_{x} \mathrm{MnO}_{3} 23$ and cobaltites $\mathrm{LaCoO}_{3} 24$.

The LDA+GTB may be considered as the straightforward development of the Hubbard atomic representation approach 4 to real materials like $3 d$ metal oxides 25 . Indeed, the GTB is a specific version of cluster perturbation theory (CPT) in the Hubbard $X$-operators representation 26 .

Later we are going to describe the method and its applications to three classes of materials with SEC: cuprates, manganities and cobaltites. The rest of the Review is organized as follows. In the next Section we provide the main ideas and technical steps of the LDA+GTB and consider different approximations to solve the Dyson equation in the $X$-representation. In Section 3 we discuss the LDA+GTB band structure of $\mathrm{La}_{2-x} \mathrm{Sr}_{x} \mathrm{CuO}_{4}$. Section 4 and Section 5 are devoted to manganites and cobaltites, respectively. Section 6 is the conclusion.

\section{LDA+GTB method}

The very first step in deriving the GTB method is to define an "electron" in system with SEC. Due to the strong interactions the free electrons are so heavily renormalized, so that the new objects are unlikely to be called "the electrons". Still, we would like to generate the output of the theory in terms of the Green function that is the one-particle property, so the "electron Green function". The goal is to find the electron Green function or, in other words, to define the electron. According to the exact Lehmann representation 27 , at $T=0$ the electron Green function can be 
written as

$$
G_{\sigma}(\vec{k}, \omega)=\sum_{m}\left(\frac{A_{m}(\vec{k}, \omega)}{\omega-\Omega_{m}^{+}}+\frac{B_{m}(\vec{k}, \omega)}{\omega-\Omega_{m}^{-}}\right) .
$$

where $\Omega_{m}^{+}=E_{m}(N+1)-E_{0}(N)-\mu, \Omega_{m}^{-}=E_{0}(N)-E_{m}(N-1)-\mu, \mu$ is the chemical potential, and numerators are equal to

$$
\begin{aligned}
& A_{m}(\vec{k}, \omega)=\left|\left\langle 0, N\left|a_{\vec{k} \sigma}\right| m, N+1\right\rangle\right|^{2}, \\
& B_{m}(\vec{k}, \omega)=\left|\left\langle m, N-1\left|a_{\vec{k} \sigma}\right| 0, N\right\rangle\right|^{2} .
\end{aligned}
$$

Here, $|m, N\rangle$ is the $m$-th eigenstate of the $N$ electron system, $H|m, N\rangle=$ $E_{m}|m, N\rangle$.

Since each single pole contribution on the right-hand side of Eq. (11) corresponds to some QP, we interpret the Lehmann representation in the following way: electron is the linear superposition of QPs with the energies $\Omega_{m}^{+}\left(\Omega_{m}^{-}\right)$for electron addition (removal) and with the spectral weights $A_{m}\left(B_{m}\right)$. At finite temperatures, the Lehmann representation determines the QP as the excitation between two arbitrary $|m, N+1\rangle$ and $|n, N\rangle$ terms with the energy $\Omega_{m n}=E_{m}-E_{n}$ and a temperature dependent spectral weight 27 . This definition is very clear. Unfortunately in general case it cannot be used straightforwardly because the exact eigenstates $|m, N\rangle$ and the eigenenergies $E_{m}$ are unknown. The Landau Fermi-liquid QP is a specific case of the Eq. (11) with only one QP close to the Fermi level. For the free electron with energy $\varepsilon_{0}$, all QP energies are equal to $\Omega_{m}^{+}=\Omega_{m}^{-}=\varepsilon_{0}-\mu$. Now we are going to show that the GTB method is the perturbative realization of the Lehmann representation.

As any other CPT approach, the GTB method starts with the exact diagonalization (ED) of the intracell part $\left(H_{c}\right)$ of the multielectron Hamiltonian and treats the intercell part $\left(H_{c c}\right)$ by a perturbation theory. Thus we make a realization of the Lehmann representation inside one unit cell with all local QP energies and spectral weights calculated via ED. The total LDA + GTB procedure consists of the following steps 22 :

Step I: LDA. Calculation of the LDA band structure, construction of Wannier functions with the given symmetry, and computation of the one- and twoelectron matrix elements of the TB Hamiltonian with the local and nearestneighbor Coulomb interactions.

Step II: ED. Separation of the total Hamiltonian $H$ into the intra- and inter-cell parts, $H=H_{c}+H_{c c}$, where $H_{c}$ represents the sum of the orthogonal unit cells, $H_{c}=\sum_{f} H_{f}$. ED of a single unit cell term, $H_{f}$, and construction of the Hubbard $X$-operators Hubbard $X$-operators $X_{f}^{p q}=|p\rangle\langle q|$ using the complete orthogonal set of eigenstates $\{|p\rangle\}$ of $H_{f}$.

Step III: Perturbation theory. Within the $X$-representation, local interactions are diagonal and all intercell hoppings and long-range Coulomb interaction terms have the bilinear form in the $X$-operators. Various perturbation 
approaches known for the Hubbard model in the $X$-representation can be used. The most general one includes treatment within the generalized Dyson equation obtained by the diagram technique 26 .

Below we discuss each step in detail.

\subsection{Step I: LDA}

LDA provides us a set of Bloch functions $\left|\Psi_{\lambda \vec{k}}\right\rangle$ ( $\lambda$ is band index) and band energies $\varepsilon_{\lambda}(\vec{k})$. For example, LDA band structure calculation for $\mathrm{La}_{2} \mathrm{CuO}_{4}$ and $\mathrm{Nd}_{2} \mathrm{CuO}_{4}$ was done within the TB-LMTO-ASA (linear muffin-tin orbitals using atomic sphere approximation in the tight-binding) method 28 . Using the Wannier functions ( $W$ Fs) formalism 29 or the NMTO method $\frac{30}{20}$, we obtain single electron energies $\varepsilon_{\lambda}$ and hopping integrals $T_{f g}^{\lambda \lambda^{\prime}}$ of the TB mode $22 \mid 31$

$$
\begin{aligned}
H & =\sum_{f, \lambda, \sigma}\left(\epsilon_{\lambda}-\mu\right) n_{f \lambda \sigma}+\sum_{f \neq g} \sum_{\lambda, \lambda^{\prime}, \sigma} T_{f g}^{\lambda \lambda^{\prime}} c_{f \lambda \sigma}^{\dagger} c_{g \lambda^{\prime} \sigma} \\
& +\frac{1}{2} \sum_{f, g, \lambda, \lambda^{\prime} \sigma_{1}, \sigma_{2}, \sigma_{3}, \sigma_{4}} V_{f g}^{\lambda \lambda^{\prime}} c_{f \lambda \sigma_{1}}^{\dagger} c_{f \lambda \sigma_{3}} c_{g \lambda^{\prime} \sigma_{2}}^{\dagger} c_{g \lambda^{\prime} \sigma_{4}},
\end{aligned}
$$

where $c_{f \lambda \sigma}$ is the annihilation operator in the Wannier representation of the hole at the site $f$ on the orbital $\lambda$ and with the spin $\sigma, n_{f \lambda \sigma}=c_{f \lambda \sigma}^{\dagger} c_{f \lambda \sigma}$. Note that a number and a symmetry of chosen $W F s$ are determined by the energy window that we are interested in.

The values of Coulomb parameters $V_{f g}^{\lambda \lambda^{\prime}}$ are obtained by LDA supercell calculations 32 . For $\mathrm{Cu}$ in $\mathrm{La}_{2} \mathrm{CuO}_{4}$, Hubbard parameter $U$ and Hund's exchange $J_{H}$ are equal to $10 \mathrm{eV}$ and $1 \mathrm{eV}$, respectively 33 .

\subsection{Step II: Exact Diagonalization}

In transition metal (Me) oxides, the unit cell may be chosen as the $\mathrm{MeO}_{n}(n=$ $6,5,4)$ cluster and usually there is a common oxygen shared by two adjacent cells. All other ions provide the electroneutrality and contribute to the high energy electronic structure. In the low energy sector, they are inactive. Before ED calculations we solve the problem of nonorthogonality of the oxygen molecular orbitals of adjacent cells. For the $\sigma$-bonding of the $3 d$ metal $e_{g}$ electrons and $a_{1 g}, b_{1 g}$ oxygen orbitals this problem is solved explicitly using the diagonalization in $k$-space 34 . We have used the same procedure for the $t_{2 g}$ orbitals. Such orthogonalization results in the renormalization of the hopping and Coulomb matrix elements in Eq. (3). Later we will work with the renormalized parameters. After the orthogonalization, the Hamiltonian (3) can be written as a sum of intracell and intercell contributions

$$
H=H_{c}+H_{c c}, \quad H_{c}=\sum_{f} H_{f}, \quad H_{c c}=\sum_{f, g} H_{f g}
$$

with orthogonal states in different cells described by $H_{f}$. 


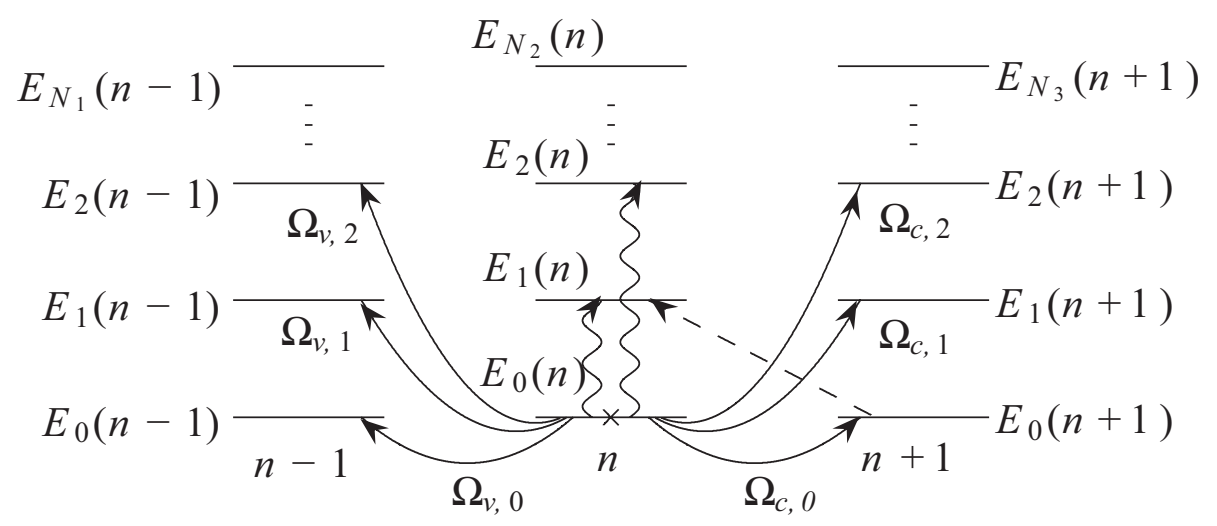

Fig. 1. The occupied ground term $E_{0}(n)$, marked by the cross, and excited terms $E_{i}(n)$ of the $d^{n}$ configuration as well as electron removal [addition] $d^{n-1}\left[d^{n+1}\right]$ sectors of the Hilbert space with energies $E_{i}(n-1)\left[E_{i}(n+1)\right]$. Vertical wavy lines show local Bose-type excitons. Solid lines with arrows show electron removal (index $\nu$ ) and electron addition (index $c$ ) Fermi-type excitations. Dashed line shows the virtual Fermi-type excitation from $E_{0}(n+1)$ to $E_{1}(n)$.

The ED of $H_{f}$ gives us the set of eigenstates $|p\rangle=\left|n_{p}, i_{p}\right\rangle$ with the energy $E_{p}$. Here, $n_{p}$ is the number of electrons per unit cell and $i_{p}$ denotes all other quantum numbers like spin, orbital moment, etc. We perform the ED with all possible excited eigenstates, not the Lancoz procedure.

How to determine which configurations are relevant? They are found from the local electroneutrality. Let's consider $\mathrm{LaMeO}_{3}$ with Me being a $3 d$ element as an example. The ionic valency is $\mathrm{La}^{3+} \mathrm{Me}^{3+} \mathrm{O}_{3}^{-2}$, thus the $3 d$ cation is $\mathrm{Me}^{3+}\left(d^{4}\right.$ for $\mathrm{Mn}^{3+}$ and $d^{6}$ for $\mathrm{Co}^{3+}$ ). Due to the covalency, the ground state of the unit cell is given by the hybridization of $d^{n} p^{6}+d^{n+1} p^{5}$ configurations (in the spectroscopic notations $d^{n}+d^{n+1} \underline{L}$, where $\underline{L}$ means a ligand hole 35 . Electron addition process results in $d^{n+1}$ subspace of the Hilbert space with mixture of $d^{n+1}+d^{n+2} \underline{L}$ configuration. Similarly, electron removal results in $d^{n-1}$ subspace with $d^{n-1}+d^{n} \underline{L}+d^{n+1} \underline{L}^{2}$ mixture. Thus for stoichiometric compound, the three relevant subspaces, $d^{n-1}$, $d^{n}$, and $d^{n+1}$, of the Hilbert space are shown in Fig. 1 For each subspace, the ED provides a set of multielectron states $|n, i\rangle$ with energy $E_{i}(n), i=0,1,2, \ldots, N_{n}$. Within this set of multielectron terms, the charge-neutral Bose-type excitations with the energy $\omega_{i}=E_{i}(n)-E_{0}(n)$ are shown by the vertical wavy lines. Electron addition excitations (local Fermi-type QP) have energies $\Omega_{c, i}=E_{i}(n+1)-E_{0}(n)$. Here, index "c" means that QPs form the empty conductivity band. Similarly, the valence band is formed by the electron removal Fermi-type QPs with energies $\Omega_{v, i}=E_{0}(n)-E_{i}(n-1)$ [a hole creation with energy $E_{i}(n-1)-E_{0}(n)$ is shown in Fig. 1. This multielectron language have been used in the spectroscopy, see for example 35 . The proper mathematical tool to study both the local QP and their 
intercell hoppings is given by the Hubbard $X$-operators ${ }^{4}$,

$$
X_{f}^{p q}=|p\rangle\langle q| .
$$

with algebra given by the multiplication rule $X_{f}^{p q} X_{f}^{r s}=\delta_{q r} X_{f}^{p s}$, and by the completeness condition $\sum_{p} X_{f}^{p p}=1$. The last two equations reflects the fact that $X$ operators are the projective operators. The operator $X_{f}^{p q}$ describes the transition from initial state $|q\rangle$ to the final state $|p\rangle, X^{p q}|q\rangle=|p\rangle$. The important property of $X$-operators is that any local operator is given by linear combination of $X$-operators. Indeed, $\hat{O}_{f}=\hat{1} \cdot \hat{O} \cdot \hat{1}=\sum_{p, q}|p\rangle\left\langle p\left|\hat{O}_{f}\right| q\right\rangle\langle q|=\sum_{p, q}\left\langle p\left|\hat{O}_{f}\right| q\right\rangle X_{f}^{p q}$. The commutation rule for Hubbard operators follows from the $X$-operators algebra and it is rather awkward. Nevertheless, if $n_{p}-n_{q}$ is odd $\left( \pm 1, \pm 3\right.$, etc.) then the $X^{p q}$ is called quasifermionic operator; if $n_{p}-n_{q}$ is even $\left(0, \pm 2\right.$, etc.) the $X^{p q}$ is a quasibosonic one 4 .

We will use a simplified notation

$$
X_{f}^{p q} \rightarrow X_{f}^{\vec{\alpha}_{m}} \rightarrow X_{f}^{m}
$$

where the number $m$ enumerate the excitation and plays the role of the QP band index; each pair $(p, q)$ corresponds to some vector $\vec{\alpha}(p, q)$ that is called "root vector" in the diagram technique ${ }^{36}$. In this notation, a single-electron (hole) creation operator is given by a linear combination in the $X$-representation, $c_{f \lambda \sigma}=\sum_{m} \gamma_{\lambda \sigma}(m) X_{f}^{m}$, $\gamma_{\lambda \sigma}(p, q)=\left\langle p\left|c_{f \lambda \sigma}\right| q\right\rangle$.

In the $X$-representation, the intracell part of the Hamiltonian is diagonal,

$$
H_{c}=\sum_{f, p}\left(E_{p}-n_{p} \mu\right) X_{f}^{p p}
$$

The intercell hopping is given by

$$
H_{c c}=\sum_{f \neq g} \sum_{m, m^{\prime}} t_{f g}^{m m^{\prime}} X_{f}^{m \dagger} X_{g}^{m^{\prime}}
$$

where the matrix elements are

$$
t_{f g}^{m m^{\prime}}=\sum_{\sigma, \lambda, \lambda^{\prime}} T_{f g}^{\lambda \lambda^{\prime}} \gamma_{\lambda \sigma}^{*}(m) \gamma_{\lambda^{\prime} \sigma}\left(m^{\prime}\right)
$$

All intraatomic $d-d$ Coulomb interactions are included in $H_{c}$ and since $H_{c}$ is diagonal in $X$-operators, they treated exactly. The dominant part of the $p-d$ and $p-p$ Coulomb interactions is also included in $H_{c}$ and gives contribution to energies $E_{p}$, while a small part of it $(\sim 10 \%)$ provides the intercell Coulomb interaction that is also bilinear in the $X$-operators, $H_{c c}^{\text {Coul. }}=\sum_{f \neq g} \sum_{p, q, p^{\prime}, q^{\prime}} V_{f g}^{p q, p^{\prime} q^{\prime}} X_{f}^{p q} X_{g}^{p^{\prime} q^{\prime}}$. 


\subsection{Step III: Perturbation Theory}

The characteristic local energy scale is given by the effective Hubbard parameter $U_{\text {eff }}=E_{0}(n+1)+E_{0}(n-1)-2 E_{0}(n)$ that is given by the difference of the initial $d^{n}+d^{n}$ and the excited $d^{n-1}+d^{n+1}$ configurations 35 . The same energy can be obtained as the local gap between the conductivity and valence bands, $U_{\text {eff }}=\Omega_{c, 0}-\Omega_{v, 0}$. Depending on the ratio of the bare Hubbard $U$ and the charge excitation energy $\Delta_{p d}=\varepsilon_{p}-\varepsilon_{d}$, the $U_{\text {eff }}$ may represent the Mott-Hubbard gap for $U<\Delta_{p d}$ or the charge transfer $(\mathrm{CT})$ gap $E_{C T}$ for $U>\Delta_{p d} 37$. The intercell hopping and interaction result in the dispersion and decrease the energy gap, $E_{g}<U_{\text {eff }}$. The intercell hoppings, Eq. (8), and the non-local Coulomb interactions can be treated by a perturbation theory. We'd like to emphasize that in the $X$-representation the perturbation, Eq. (8), has exactly the same structure as the hopping Hamiltonian in the conventional Hubbard model. That is why the accumulated experience of the Hubbard model study in the $X$-representation can be used here.

Single-electron Green function for a particle with momenta $\mathbf{k}$, energy $E$, spin $\sigma$, and orbital indices $\lambda$ and $\lambda^{\prime}, G_{\mathbf{k} \sigma}^{\lambda \lambda^{\prime}}(E) \equiv\left\langle\left\langle c_{\mathbf{k} \lambda \sigma} \mid c_{\mathbf{k} \lambda^{\prime} \sigma}^{\dagger}\right\rangle\right\rangle_{E}$, is given by a linear combination of the Hubbard operator's Green functions $D_{\mathbf{k} \sigma}^{m n}(E)=\left\langle\left\langle X_{\mathbf{k} \sigma}^{m} \mid X_{\mathbf{k} \sigma}^{n \dagger}\right\rangle\right\rangle_{E}$,

$$
G_{\mathbf{k} \sigma}^{\lambda \lambda^{\prime}}(E)=\sum_{m m^{\prime}} \gamma_{\lambda \sigma}(m) \gamma_{\lambda^{\prime} \sigma}^{*}\left(m^{\prime}\right) D_{\mathbf{k} \sigma}^{m m^{\prime}}(E) .
$$

The question is how to determine $X$-operator's Green function. One of the regular ways to treat perturbations is the diagram technique. For $X$-operators with their awkward algebra there is no standard Wick's theorem. Nevertheless, the generalized Wick's theorem has been proved quite some time agd 38 . The first convenient version of the diagram technique has been formulated in Ref. 36 . The general rules of the diagram technique for the $X$-operators are described in details in Ref. 26 , where the generalized Dyson equation has been obtained

$$
\hat{D}_{\vec{k} \sigma}(E)=\left\{\left(E-\Omega_{m}\right) \delta_{m m^{\prime}}-\hat{P}_{\vec{k} \sigma}(E) \hat{t}(\vec{k})-\hat{\Sigma}_{\vec{k} \sigma}(E)\right\}^{-1} \hat{P}_{\vec{k} \sigma}(E) .
$$

Here $\Omega_{m}=E_{p}(n+1)-E_{q}(n)$ is the $m$-th QP local energy. Besides the self-energy matrix $\hat{\Sigma}_{\mathbf{k} \sigma}(E)$, the unconventional term $\hat{P}_{\mathbf{k} \sigma}(E)$ called "the strength operator" appears due to the $X$-operators algebra. Similar term had been known for the spinoperator diagram technique 39 . This term determines the QP oscillation strength (spectral weight) as well as the renormalized bandwidth. The Hubbard I solution 4 is obtained by setting $\hat{\Sigma}_{\mathbf{k} \sigma}(E)=0$ and $\left(\hat{P}_{\mathbf{k} \sigma}(E)\right)_{m m^{\prime}}=F_{m} \delta_{m m^{\prime}}$, where $F_{m}=$ $\left\langle X^{p p}\right\rangle+\left\langle X^{q q}\right\rangle$. We call $F_{m}$ "the occupation factor"; it provides a non-zero spectral weight for the $\mathrm{QP}$ excitation between at least partially filling eigenstates and gives zero spectral weight for excitations between empty states. The dispersion equation for the QP band structure of the Hubbard fermions in this case is given by

$$
\operatorname{det}\left\|\delta_{m n}\left(E-\Omega_{m}\right) / F(m)-t^{m n}(\vec{k})\right\|=0 \text {. }
$$


The dispersion equation, Eq. (12), is similar to the conventional TB dispersion equation, but instead of a single electron local energy $\varepsilon_{\lambda}$ we have a local QP energy $\Omega_{m}$. That is why we call this approach the "generalized TB method".

The occupation numbers $\left\langle X^{p p}\right\rangle$ are calculated self-consistently via the chemical potential equation, $n_{e}=\frac{\left\langle N_{e}\right\rangle}{N}=\frac{1}{N} \sum_{f, n, i} n\left\langle X_{f}^{n, i ; n, i}\right\rangle$. The change of the concentration $n_{e}$ redistributes the occupation numbers and due to the occupation factors, $F_{m}$, changes the QP band structure.

In the GTB method, the intracell Green function can be found exactly

$$
G_{0 \sigma}^{\lambda \lambda^{\prime}}(E)=\sum_{m}\left|\gamma_{\lambda \sigma}(m)\right|^{2} \delta_{\lambda \lambda^{\prime}} D_{0 \sigma}^{m}(E)
$$

where $D_{0, \sigma}^{m}(E)=F_{m} /\left(E-\Omega_{m}+i \delta\right)$. Comparing this exact local Green function with the Lehmann representation in Eq. (11), we can say that the electron here is a linear combination of local (Hubbard) fermions with QP energy $\Omega_{m}$ and a spectral weight $\left|\gamma_{\lambda \sigma}(m)\right|^{2} F_{m}$. It is exactly the same language as in the Lehmann representation. The difference is that it is realized locally and both QP energy and spectral weight are calculated explicitly.

It should be stressed that the LDA+GTB bands are not the single electron conventional bands. There is no any single particle Schrödinger equation with the effective potential that gives the LDA+GTB band structure. These QP are excitations between different multielectron terms. The LDA+GTB bands depend on the multielectron term occupation numbers through $\hat{P}_{\mathbf{k} \sigma}(E)$ and $\hat{\Sigma}_{\mathbf{k} \sigma}(E)$ that should be calculated via the chemical potential equation. There is no rigid band behavior from the very beginning; the band structure depends on doping, temperature, pressure, and external fields.

\section{Cuprates}

In this section we present the results for cuprates with one $\mathrm{CuO}_{2}$ layer in the unit cell: hole doped cuprate $\mathrm{La}_{2-x} \mathrm{Sr}_{x} \mathrm{CuO}_{4}$. Yet there is no self-consistent treatment of both electronic and magnetic structures; our LDA+GTB calculation have been carried out for a prescribed magnetic order. Thus, we consider separately the antiferromagnetic (AFM) phase with the long-range AFM order and the spin-liquid phase with the short-range AFM correlations.

\subsection{Band Structure of the Undoped $\mathrm{La}_{2} \mathrm{CuO}_{4}$}

The ED of the multiband $p-d$ Hamiltonian (3) for the $\mathrm{CuO}_{6}$ cluster which includes the apical oxygens, results in the following local eigenstates (in the hole representation): 1) $n_{h}=0$, the vacuum state $|0\rangle$ formed by $d^{10} p^{6}$ orbital configuration, 2) $n_{h}=1$, the spin doublets $|\sigma, \lambda\rangle$ with different orbital symmetries. The lowest one is $b_{1 g},|\sigma\rangle$, and the first excited is $a_{1 g}$ molecular orbital. 3) $n_{h}=2$. A set of two-hole singlets and triplets, spread in the energy region of about $U_{d} \sim 10 \mathrm{eV}$. The lowest 


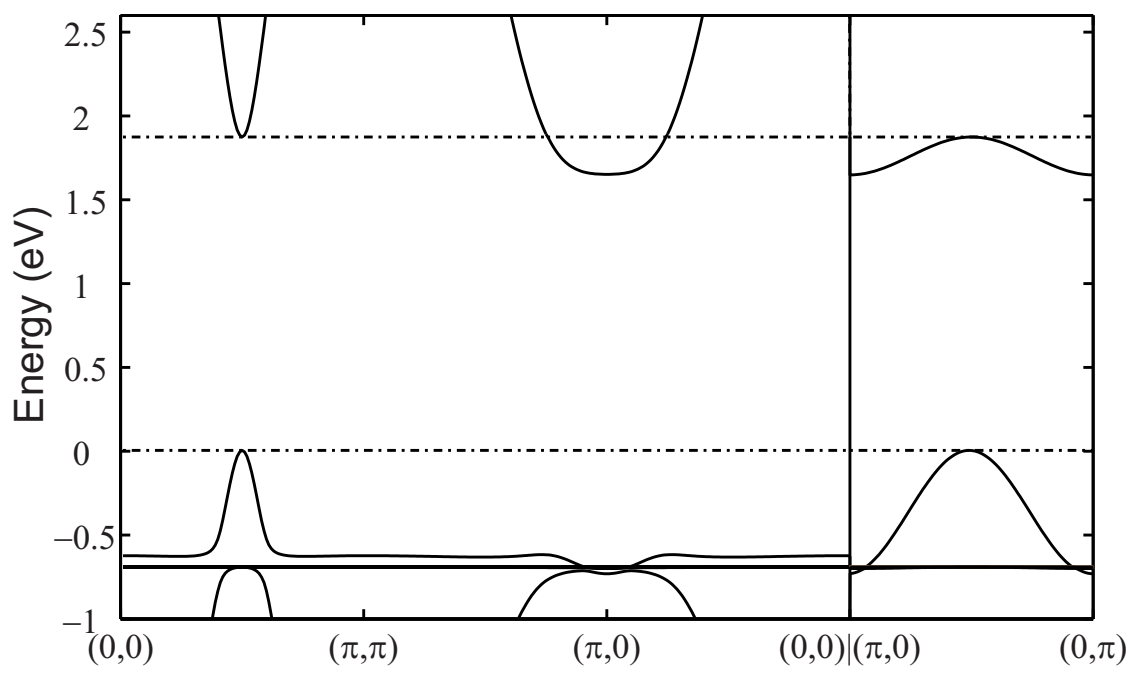

Fig. 2. The LDA + GTB band structure of the $\mathrm{AFM} \mathrm{La}_{2} \mathrm{CuO}_{4}$ along the principal cuts of the Brillouin zone 22.

one is the ${ }^{1} A_{1}$ singlet $|S\rangle$ that includes the Zhang-Rice singlet among other two-hole singlets. The first excited triplet $|T M\rangle(M=+1,0,-1)$ has the ${ }^{3} B_{1 g}$ symmetry. The total number of eigenstates is about 100 .

The next practical step is the calculation of the matrix elements, $\left\langle 0\left|c_{f \lambda \sigma}\right| 1 \sigma^{\prime} \lambda\right\rangle$, $\left\langle 1 \sigma^{\prime} \lambda\left|c_{f \lambda \sigma}\right| 2, i\right\rangle$, and construction of $X$-operators for all single electron orbitals. Here, $\lambda$ stands for $\mathrm{Cu}-d_{x^{2}-y^{2}}, \mathrm{Cu}-d_{z^{2}}$, O- $b$, O- $a$, or O- $p_{z}$ orbital. For the AFMordered $\mathrm{La}_{2} \mathrm{CuO}_{4}$, we use the two-sublattice $(A$ and $B)$ version of the Hubbard $\mathrm{I}$ solution with two occupation factors, $F_{m, A}$ and $F_{m, B}$. Due to the effective molecular field, the local $b_{1 g}$ spin doublet is splitted so that at $T=0:\left\langle X_{A}^{\uparrow \uparrow}\right\rangle=\left\langle X_{B}^{\downarrow \downarrow}\right\rangle=$ $1,\left\langle X_{A}^{\downarrow \downarrow}\right\rangle=\left\langle X_{B}^{\uparrow \uparrow}\right\rangle=0$. The GTB band structure and the DOS in the wide energy region with all excited two-hole states $|2, i\rangle$ have been calculated in Ref. 40 . The empty conductivity band is formed by only one Hubbard fermion, $X_{f}^{0, \sigma}$. It is separated by the CT gap $U_{\text {eff }}=E_{C T} \approx 2 \mathrm{eV}$ from the filled valence band. The valence band is formed by a large number of Hubbard fermions $X_{f}^{\sigma, 2 i}$ and consists of a set of narrow bands with the total bandwidth about $6 \mathrm{eV}$. If we are interested in a smaller energy window around the $E_{C T}$ (for example, to study ARPES), it is possible to simplify the calculation by neglecting the high-energy states from both $|2, i\rangle$ and $\left|1 \sigma^{\prime} \lambda\right\rangle$ sets. Then the minimal realistic basis is $\{|0\rangle,|\sigma\rangle,|S\rangle,|T M\rangle\}$.

The $X$-representation for the fermionic operators in this basis is $c_{f d_{x^{2}-y^{2}} \sigma}=$ $u X_{f}^{0 \sigma}+2 \sigma \gamma_{x} X_{f}^{\bar{\sigma} S}, c_{f p_{b} \sigma}=v X_{f}^{0 \sigma}+2 \sigma \gamma_{b} X_{f}^{\bar{\sigma} S}, c_{f p_{a} \sigma}=\gamma_{a}\left(\sigma \sqrt{2} X_{f}^{\bar{\sigma} T 0}-X_{f}^{\sigma T 2 \sigma}\right)$, $c_{f d_{z^{2}} \sigma}=\gamma_{z}\left(\sigma \sqrt{2} X_{f}^{\bar{\sigma} T 0}-X_{f}^{\sigma T 2 \sigma}\right), c_{f p_{z} \sigma}=\gamma_{p}\left(\sigma \sqrt{2} X_{f}^{\bar{\sigma} T 0}-X_{f}^{\sigma T 2 \sigma}\right)$. Here, $\bar{\sigma} \equiv-\sigma$ and $T 2 \sigma$ stands for $T(+1)$ or $T(-1)$ depending on the value of the spin label 
$\sigma= \pm 1 / 2$. The explicit form of the TB Hamiltonian (3) in this basis looks like the two-band singlet-triplet Hubbard model:

$$
\begin{aligned}
H_{p d} & =\sum_{f}\left[\varepsilon_{1} \sum_{\sigma} X_{f}^{\sigma \sigma}+\varepsilon_{2 S} X_{f}^{S S}+\varepsilon_{2 T} \sum_{M} X_{f}^{T M T M}\right] \\
& +\sum_{f \neq g, \sigma}\left[t_{f g}^{00} X_{f}^{\sigma 0} X_{g}^{0 \sigma}+t_{f g}^{S S} X_{f}^{S \bar{\sigma}} X_{g}^{\bar{\sigma} S}+2 \sigma t_{f g}^{0 S}\left(X_{f}^{\sigma 0} X_{g}^{\bar{\sigma} S}+h . c .\right)\right. \\
& +t_{f g}^{S T}\left\{\left(\sigma \sqrt{2} X_{f}^{T 0 \bar{\sigma}}-X_{f}^{T 2 \sigma \sigma}\right)\left(v X_{g}^{0 \sigma}+2 \sigma \gamma_{b} X_{g}^{\bar{\sigma} S}\right)+\text { h.c. }\right\} \\
& \left.+t_{f g}^{T T}\left(\sigma \sqrt{2} X_{f}^{T 0 \bar{\sigma}}-X_{f}^{T 2 \sigma \sigma}\right)\left(\sigma \sqrt{2} X_{g}^{\bar{\sigma} T 0}-X_{g}^{\sigma T 2 \sigma}\right)\right]
\end{aligned}
$$

The hopping parameters, Eq. (9), of the effective Hubbard model are expressed through the microscopic ab initio parameters $t_{p d}$ and $t_{p p}, t_{f g}^{00}=-2 t_{p d} \mu_{f g} 2 u v-$ $2 t_{p p} \nu_{f g} v^{2}, t_{f g}^{S S}=-2 t_{p d} \mu_{f g} 2 \gamma_{x} \gamma_{b}-2 t_{p p} \nu_{f g} \gamma_{b}^{2}, t_{f g}^{0 S}=-2 t_{p d} \mu_{f g}\left(v \gamma_{x}+u \gamma_{b}\right)-$ $2 t_{p p} \nu_{f g} v \gamma_{b}, t_{f g}^{T T}=\frac{2}{\sqrt{3}} t_{p d} \lambda_{f g} 2 \gamma_{a} \gamma_{z}+2 t_{p p} \nu_{f g} \gamma_{a}^{2}-2 t_{p p}^{\prime} \lambda_{f g} 2 \gamma_{p} \gamma_{a}, t_{f g}^{S T}=\frac{2}{\sqrt{3}} t_{p d} \xi_{f g} \gamma_{z}+$ $2 t_{p p} \chi_{f g} \gamma_{a}-2 t_{p p}^{\prime} \xi_{f g} \gamma_{p}$. Here $\mu_{f g}, \nu_{f g}, \lambda_{f g}, \xi_{f g}$, and $\chi_{f g}$ are the coefficients of the oxygen group orbitals construction, and $u, v, \gamma_{x}, \gamma_{b}, \gamma_{a}, \gamma_{p}$, and $\gamma_{z}$ are the matrix elements $\gamma_{\lambda \sigma}(p, q)$ (see Ref. 20 for details).

The QP band structure of $\mathrm{La}_{2} \mathrm{CuO}_{4}$ is shown in Fig. 2 for the $\Gamma(0,0)-M(\pi, \pi)-$ $X(\pi, 0)-\Gamma(0,0)$ and $X(\pi, 0)-Y(0, \pi)$ cuts of the square lattice Brillouin zone. Zero at the energy scale is not the Fermi level but rather fixed by the condition $\varepsilon_{d_{x^{2}-y^{2}}}=0$.

The top of the valence band is at the $\bar{M}=(\pi / 2, \pi / 2)$ point, while the bottom of the conductivity band is at the $X$ point. The dispersion of the valence band determined by the hybridization of the two bands which formed by either $X_{f}^{\bar{\sigma} S}$ or $X_{f}^{\bar{\sigma} T M}$ Hubbard fermions. The hybridization between them is provided by the $t^{S T}$ hopping matrix elements in Eq. (14). These are fermionic bands, but frequently in the literature terms "singlet band" and "triplet band" are used. These terms reflect the final two-hole states involved in the QP excitations. The dominant spectral weight in the singlet band stems from the oxygen $b_{1 g}$ states, while for the bottom of the conductivity band it is from the $d_{x^{2}-y^{2}}$ states of $\mathrm{Cu}$.

\subsection{Cascade of Lifshitz Quantum Phase Transitions in $\mathrm{La}_{2-x} \mathrm{Sr}_{x} \mathrm{CuO}_{4}$}

The low-energy Hamiltonian for $\mathrm{La}_{2-x} \mathrm{Sr}_{x} \mathrm{CuO}_{4}$ (LSCO) is the $t-t^{\prime}-t^{\prime \prime}-J^{*}$ model obtained via exclusion of the interband (through the charge-transfer gap) excitations. Here $J^{*}$ means that besides the Heisenberg exchange term a three-site 
correlated hopping $H_{3}$ is also included, $H_{t-J^{*}}=H_{t J}+H_{3}$, where

$$
\begin{aligned}
H_{t J} & =\sum_{f, \sigma}(\varepsilon-\mu) X_{f}^{\sigma \sigma}+\sum_{f} 2(\varepsilon-\mu) X_{f}^{S S} \\
& +\sum_{f \neq g, \sigma}\left[t_{f g} X_{f}^{S \bar{\sigma}} X_{g}^{\bar{\sigma} S}+\frac{J_{f g}}{4}\left(X_{f}^{\sigma \bar{\sigma}} X_{g}^{\bar{\sigma} \sigma}-X_{f}^{\sigma \sigma} X_{g}^{\bar{\sigma} \bar{\sigma}}\right)\right], \\
H_{3} & =\sum_{f \neq m \neq g, \sigma} \frac{\tilde{t}_{f m} \tilde{t}_{m g}}{U_{e f f}}\left(X_{f}^{\sigma S} X_{m}^{\bar{\sigma} \sigma} X_{g}^{S \bar{\sigma}}-X_{f}^{\sigma S} X_{m}^{\bar{\sigma} \bar{\sigma}} X_{g}^{S \sigma}\right) .
\end{aligned}
$$

Here $t_{f g}=t_{f g}^{S S}$ is the hopping in the UHB, $J_{f g}=\tilde{t}_{f g}^{2} / U_{e f f}$ is the exchange interaction due to the interband (UHB $\leftrightarrow$ LHB) hopping $\tilde{t}_{f g}=t_{f g}^{0 S}$ through the charge-transfer gap $U_{\text {eff }}=E_{C T}$, hole creation operator is now $\tilde{a}_{f \sigma}^{\dagger}=2 \sigma X_{f}^{S \bar{\sigma}}$ and its algebra is different from the bare fermion's one. The spin operators are also easily expressed via $X$-operators, $S_{f}^{+}=X_{f}^{\sigma \bar{\sigma}}, S_{f}^{z}=\left(X_{f}^{\sigma \sigma}-X_{f}^{\bar{\sigma} \bar{\sigma}}\right) / 2$.

Here we dropped out the triplet state $|T M\rangle$ because the triplet itself and the singlet-triplet excitations do not contribute to the near-Fermi level physics.

The $a b$ initio derived parameters for LSCO are (in $\mathrm{eV}) t=0.93, t^{\prime}=-0.12$, $t^{\prime \prime}=0.15, \tilde{t}=0.77, \tilde{t}^{\prime}=-0.08, \tilde{t}^{\prime \prime}=0.12, J=0.29, J^{\prime}=0.003, J^{\prime \prime}=0.007$.

Our approach is essentially a perturbation theory with the small parameter $t / U$ contrary to the usual Fermi liquid perturbation expansion in terms of $U$ which is large in cuprates. We use a method of irreducible Green functions which is similar to the Mori-type projection technique, with the zero-order Green function given by the well-known Hubbard I approximation. Beyond it there are spin fluctuations. To provide a description of them, the self-energy was calculated in the non-crossing approximation by neglecting vertex renormalization that is equivalent to the selfconsistent Born approximation (SCBA) 41 . Resulting electron self-energy contains the space-time dependent spin correlation function $C(\mathbf{q}, \omega)$ and results in the finite quasiparticle lifetime, $\operatorname{Im} \Sigma(\mathbf{k}, \omega) \neq 0$. Note that at low temperatures $T \leq 10 \mathrm{~K}$ the spin dynamics is much slower than the electron one. A typical spin fluctuation time, $10^{-9} \mathrm{sec}$, is much larger than the electronic time $10^{-13} \sec \frac{42}{}$; that is why we can safely neglect the time dependence of the spin correlation function, $C(\mathbf{q}, \omega) \rightarrow C_{\mathbf{q}}$. The self-energy becomes static, $\Sigma(\mathbf{k}, \omega) \rightarrow \Sigma(\mathbf{k})$, and we have $\operatorname{Im} \Sigma=0$. Note that $\Sigma(\mathbf{k}, \omega)$ here is the object completely different from the one in the Fermi liquid approach because the here it is build by the diagrams for the $X$-operators, not the standard Fermionic annihilation-creation operators $a_{f \sigma}$. In the usual Fermi liquid expansion dynamical self-energy definitely plays a crucial role in the lightly doped cuprates. Here, our theory starts from a different limit where the lowest order approximation is represented by the Hubbard I solution. The corrections to the strongly-correlated mean-field approach are small because the starting point is already a reasonable approximation for the Mott-Hubbard insulator. That is proved by the small effect of the frequency dependence of the self-energy in Refs. 41143 . Moreover the doping-dependence of the FS is determined by $\operatorname{Re} \Sigma$, and it is qualitatively similar in our approach $\frac{44}{4}$ and in the approach which properly takes $\operatorname{Im} \Sigma$ 
into account $41 \mid 43$.

The vertex corrections to the self-energy are small far from the spin-density wave or the charge-density wave instabilities, that is true for the moderate doping. Our approximation for the self-energy is done in the framework of the mode-coupling approximation which has been proved to be quite reliable even for systems with strong interaction $45 \mid 46$. As shown in the spin-polaron treatment of the $t-J$ model, the vertex corrections to the non-crossing approximation are small and give only numerical renormalization of the model parameters 47 .

Green function $\left\langle\left\langle X_{\mathbf{k}}^{\bar{\sigma} S} \mid X_{\mathbf{k}}^{S \bar{\sigma}}\right\rangle\right\rangle_{\omega}$ for a hole moving on the background of shortrange AFM order is

$$
G(\mathbf{k}, \omega)=\frac{(1+x) / 2}{\omega-\varepsilon+\mu-\frac{1+x}{2} t_{\mathbf{k}}-\frac{1-x^{2}}{4} \frac{\tilde{t}_{\mathbf{k}}^{2}}{U_{e f f}}+\Sigma(\mathbf{k})},
$$

where

$$
\begin{aligned}
\Sigma(\mathbf{k}) & =-\frac{2}{1+x} \frac{1}{N} \sum_{\mathbf{q}}\left\{\left[t_{\mathbf{k}-\mathbf{q}}-\frac{1-x}{2} J_{\mathbf{q}}+\frac{1-x}{2} \frac{\tilde{t}_{\mathbf{k}-\mathbf{q}}^{2}}{U_{\text {eff }}}\right.\right. \\
& \left.\left.-\frac{1+x}{2} \frac{2 \tilde{t}_{\mathbf{k}} \tilde{t}_{\mathbf{k}-\mathbf{q}}}{U_{\text {eff }}}\right]\left(\frac{3}{2} C_{\mathbf{q}}+K_{\mathbf{k}-\mathbf{q}}\right)-\frac{1+x}{2} \frac{\tilde{t}_{\mathbf{q}}^{2}}{U_{\text {eff }}} K_{\mathbf{q}}\right\} .
\end{aligned}
$$

Here, $t_{\mathbf{k}}$ and $\tilde{t}_{\mathbf{k}}$ are the Fourier transforms of hoppings $t_{f g}$ and $\tilde{t}_{f g}$, respectively. The self-energy is determined by static spin correlation function $C_{0 n}=\left\langle S_{0}^{+} S_{n}^{-}\right\rangle$ and kinetic correlation function $K_{0 n}=\sum_{\sigma}\left\langle\tilde{a}_{0 \sigma}^{\dagger} \tilde{a}_{n \sigma}\right\rangle$ between sites 0 and $n$. These correlation functions and their Fourier transforms $C_{\mathbf{q}}$ and $K_{\mathbf{q}}$ represent the AFM short-range order and the valence-bond order, respectively. In contrast to approach of Ref. 41, we calculate these correlation functions self-consistently up to $n=9$ (ninth coordination sphere) together with the chemical potential $\mu$. To get the spin correlation function we also obtain the spin Green function $\left\langle\left\langle X_{\mathbf{q}}^{\sigma \bar{\sigma}} \mid X_{\mathbf{q}}^{\bar{\sigma} \sigma}\right\rangle\right\rangle_{\omega}$ in a spherically-symmetric spin liquid state 48449 with $\left\langle S^{z}\right\rangle=0$ and the equal correlation functions for each spin component, $\left\langle S_{0}^{+} S_{n}^{-}\right\rangle=2\left\langle S_{0}^{z} S_{n}^{z}\right\rangle=C_{0 n}$. Both $C_{0 n}$ and $K_{0 n}$ are essentially doping-dependent and $C_{0 n}$ decrease with the doping 44 . While the nearest neighbor function $C_{01}$ is finite for all studied $x$ up to $x=0.4$ with a kink at $x=p^{*}=0.24$, more distant spin correlations fall down to zero for $x>p^{*}$.

The calculated FS twice changes its topology with doping 44, see Fig. 3. Small hole pockets around $( \pm \pi / 2, \pm \pi / 2)$ points are present at small doping; then they increase in size and touch each other in the non-symmetric points $\mathbf{k}= \pm \pi(1, \pm 0.4)$ at $x_{c 1}=p_{\text {opt }}=0.151$. Above $p_{\text {opt }}$, there are two FSs around $(\pi, \pi)$ with outer being a hole-like and inner being an electron-like. The electron FS collapsed at $x_{c 2}=p^{*}=0.246$, and at $x>p^{*}$ we have only one large hole surface around $(\pi, \pi)$. Similar conclusion on the coexistence of hole and electron FS at some intermediate 

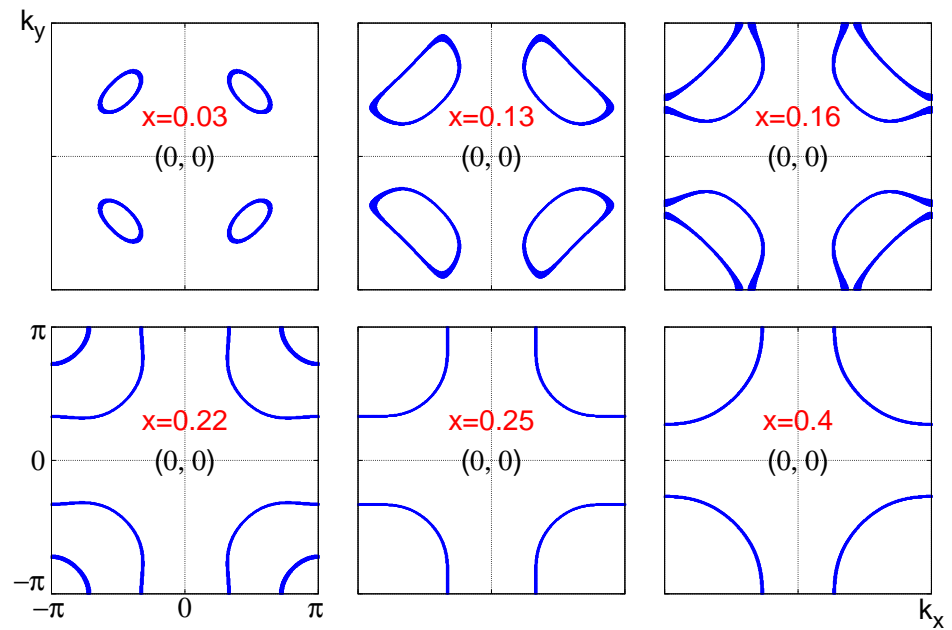

Fig. 3. (Color online) Mean-field Fermi surface transitions with doping $x$ as calculated from poles of Eq. (15). There are two topological changes: first one between $x=0.13$ and 0.16 , and second

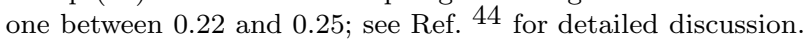

doping have been also drawn recently $\stackrel{50 \mid 51}{ }$, and earlier for the spin-density wave sate of the Hubbard model 52 .

From this consideration it follows that the FS topological transitions in cuprates are induced by doping and they are due to the non-rigid band behavior of the quasiparticles in systems with SEC. According to the general Lifshitz analysis 53 for the three dimensional (3D) system, a change of topology at the energy $\varepsilon=\varepsilon_{c}$ either by appearance of a new segment (like we found at $p^{*}$ ) or by change of its connectivity (like at $p_{\text {opt }}$ ) would result in the additional DOS, $\delta N(\varepsilon) \sim\left(\varepsilon-\varepsilon_{c}\right)^{1 / 2}$, and the change in the thermodynamic potential, $\delta \Omega \sim\left(\varepsilon_{F}-\varepsilon_{c}\right)^{5 / 2}$ (the QPT of the 2.5 -order), where $\varepsilon_{F}$ is the Fermi energy. However, due to the strong anisotropy of electronic and magnetic properties, cuprates are quasi-2D and not isotropic 3D systems. The electron hopping perpendicular to the $\mathrm{CuO}_{2}$ layers in a single-layer cuprates like LSCO is negligibly small.

The change of the FS topology at $x_{c 1}=p_{\text {opt }}$ results in the logarithmic divergence of DOS, while the emergence of the new electron-like pocket below $x_{c 2}=p^{*}$ results in a step in DOS (Fig. (4). The total DOS is a sum of the singular and regular contributions. We would like to stress that both logarithmic and step DOS singularities are in perfect agreement with the general properties of the van Hole singularities for the $2 \mathrm{D}$ electrons 54 . Contrary to the $3 \mathrm{D}$ systems, the thermodynamical potential for the $2 \mathrm{D}$ electrons has a singular contribution $\delta \Omega \sim\left(\varepsilon_{F}-\varepsilon_{c}\right)^{2}$ for the step singularity and $\delta \Omega \sim\left(\varepsilon_{F}-\varepsilon_{c}\right)^{2} \ln \left|\varepsilon_{F}-\varepsilon_{c}\right|$ for the logarithmic singularity 5 . Thus QPT at $x_{c 2}=p^{*}$ is of the second order, while at $x_{c 1}=p_{\text {opt }}$ the singularity is stronger. It is immediately follows that the Sommerfeld parameter $\gamma$ in the 

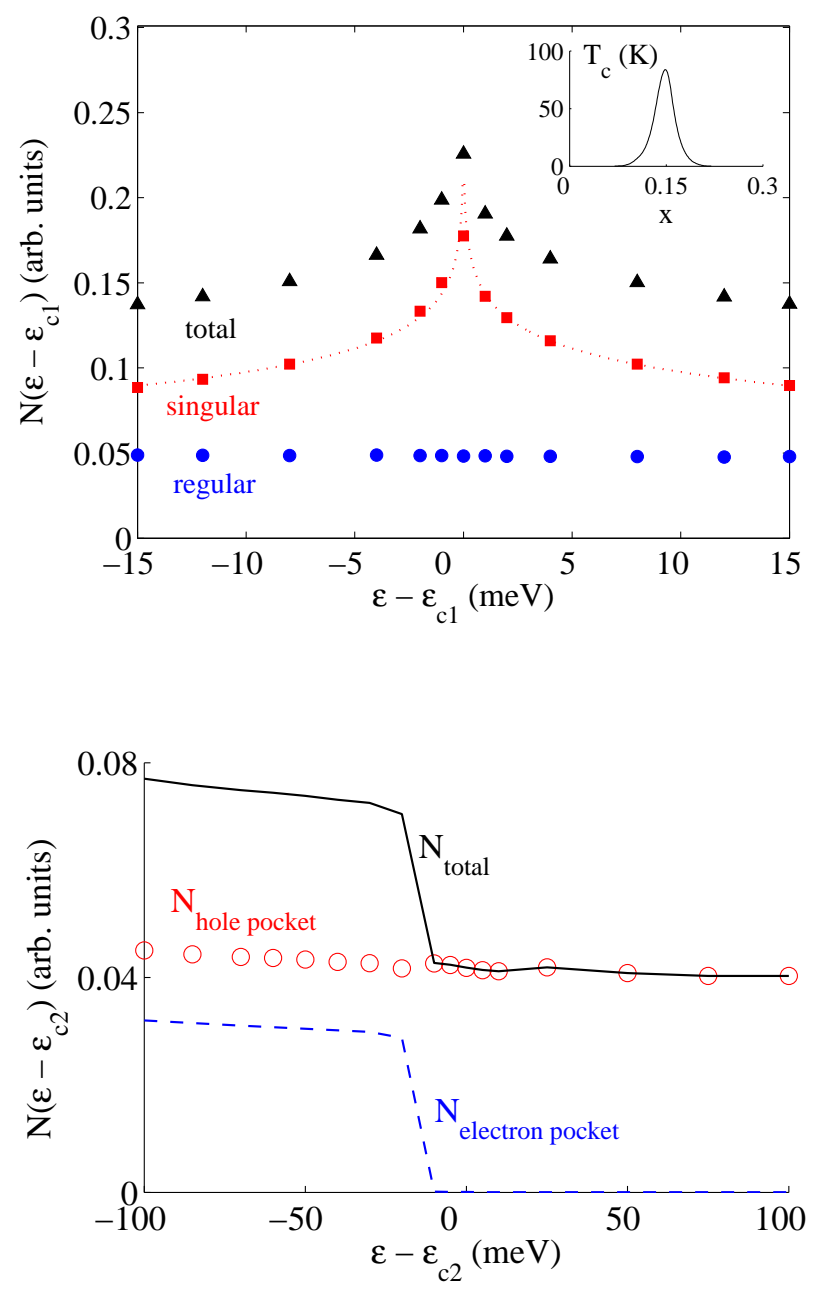

Fig. 4. Regular, singular, and total density of states $N\left(\varepsilon-\varepsilon_{c i}\right)$ near the optimal doping $\varepsilon_{c 1}=$ $\varepsilon_{F}\left(p_{o p t}\right)$ [top] and near the pseudogap critical point $\varepsilon_{c 2}=\varepsilon_{F}\left(p^{*}\right)$ [bottom], as calculated from the Green function (15). Dotted curve shows the logarithmic fitting. Below $p^{*}=0.24\left(\varepsilon<\varepsilon_{c 2}\right)$ a singular step-like contribution to the total DOS appears due to the appearance of the electron pocket. In the inset, the doping dependence of the superconducting critical temperature $T_{c}(x)$ is shown; the optimal doping is 0.151 . Note that the energy $\varepsilon-\varepsilon_{c 1}$ is the energy of holes.

electronic heat capacity $\gamma=C_{e} / T$ has also a singular step contribution at $x \leq p^{*}$, and $\delta \gamma \propto \ln \left(\varepsilon_{F}-\varepsilon_{c}\right) \propto \ln \left|x-x_{o p t}\right|$ near $x_{c 1}=p_{o p t}$. Similar divergence in the specific heat was found within the dynamical cluster approximation for the Hubbard model 56 . 
The coincidence of $x_{c 1}$ with $p_{\text {opt }}$ and $x_{c 2}$ with $p^{*}$ is not occasional. We compare the superconducting critical temperature dependence $T_{c}(x)$ in the same mode 57 as a function of doping and observe that $T_{c}(x)$ has a maximum at $x_{o p t}$ (see inset in Fig. (4), which indeed equals to $x_{c 1}$. It is not a coincidence since like in the BCS theory the maximum in $T_{c}(x)$ is determined by the maximum DOS, and at $x_{c 1}$ we have a logarithmic singularity. Kinetic energy, $E_{k i n}=\sum_{n} t_{0 n} K_{0 n}$, reveals a remarkable kink at $x_{c 2}=p^{*}$ due to the change in DOS. Above $p^{*}, E_{k i n}(p) / E_{k i n}\left(p^{*}\right) \sim 1+p$ that is expected for a conventional $2 \mathrm{D}$ metal with the hole concentration $n_{h}=1+p$ and $E_{k i n} \sim \varepsilon_{F} \sim n_{h}$. The extrapolation of this law below $p^{*}$ reveals that actual $E_{k i n}$ is much smaller. We associate this depletion with the pseudogap formation and it fits very well with the Loram-Cooper mode $[58 \mid 59$ - a simple free electron gas with a triangular pseudogap DOS,

$$
N(\varepsilon)=\left\{\begin{array}{l}
g,\left|\varepsilon-\varepsilon_{F}\right|>E_{g} \\
g \frac{\left|\varepsilon-\varepsilon_{F}\right|}{E_{g}},\left|\varepsilon-\varepsilon_{F}\right|<E_{g} .
\end{array}\right.
$$

Here $E_{g}=J\left(p^{*}-p\right) / p^{*}$ is a doping dependent pseudogap and $J$ is the nearestneighbor exchange parameter. The observed depletion of the kinetic energy together with the jump in DOS relates the QPT at $x_{c 2}$ to the pseudogap and the coincidence of $x_{c 2}$ and $p^{*}$ is not occasional.

The energy dependence of the electron self-energy is crucial and determines the Mott-Hubbard transition in the Hubbard model as was convincingly demonstrated by the dynamical mean-field theory (DMFT) 60 . Cluster generalization of DMFT 14 15 16 17 is necessary to study electron correlations in a two-dimensional $\mathrm{CuO}_{2}$ layer where the nearest neighbor spin correlations require the momentum dependent self-energy. The cellular DMFT (CDMFT) method provides $k$-dependent self-energy and results in the phase diagrams that have features similar to the ones experimentally observed in cuprates 61|62|63|64|65]. Recently, the exact diagonalization version of CDMFT (CDMFT+ED) was used to study the electronic structure of the doped Mott-Hubbard insulator $66 \mid 67$. The sequence of the FS transforma-

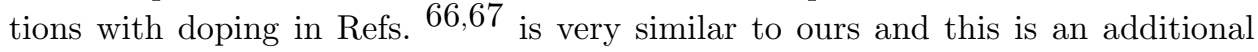
prove of the our approach's validity at least at low temperatures and away from the Fermi-liquid regime.

\section{Band Structure of Manganite $\mathrm{La}_{1-x} \mathrm{Sr}_{x} \mathrm{MnO}_{3}$}

The most unusual solutions of Eq. (12) are those with the zero spectral weight $\left(F_{m}=0\right)$. We know the QP energy and this QP has zero number of states. We call such QP the "virtual QP". To obtain the non-zero spectral weight $F_{m} \neq 0$ the non-zero occupation of the initial or final states for the excitation $\alpha_{m}=(p, q)$ is required. It may be achieved by doping, pressure, finite temperature, and external field. The virtual states have been found in GTB calculations for $\mathrm{La}_{2} \mathrm{CuO}_{4}{ }^{20}$ and for $\mathrm{FeBO}_{3} 68$. Recently they have been experimentally observed in $\mathrm{FeBO}_{3}$ by the IR (infra-red) spectroscopy 69 . 

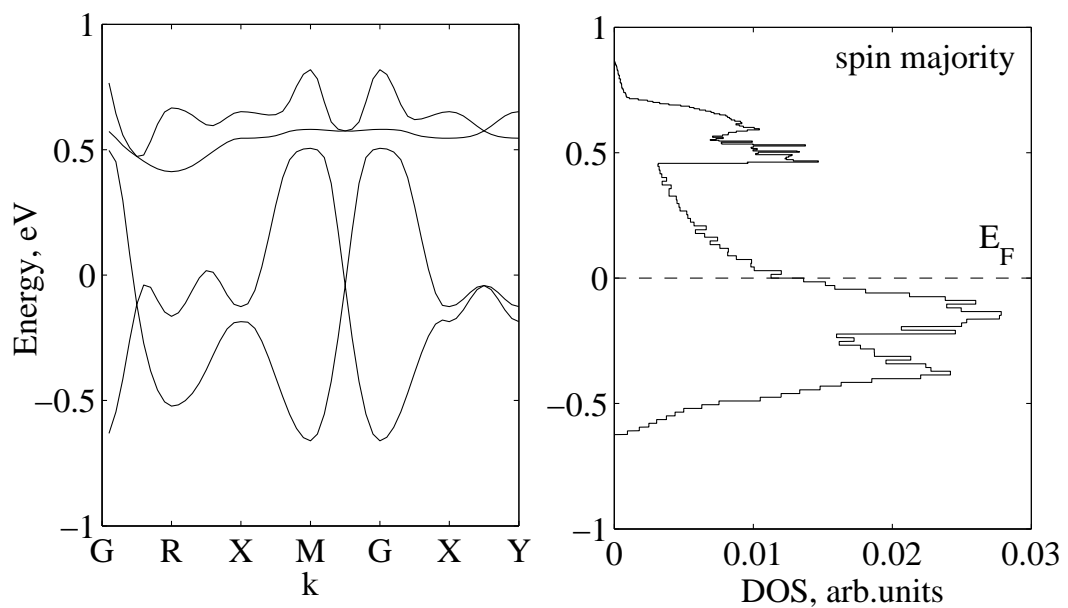

Fig. 5. LDA+GTB dispersion of the in-gap and valence bands (on the left) and spin majority density of states (on the right) for $\mathrm{La}_{1-x} \mathrm{Sr}_{x} \mathrm{MnO}_{3}$ in the ferromagnetic phase $(x=0.3)$. The spin minority DOS in the same energy region is zero. Here, $G=(0,0,0), R=(\pi, \pi, \pi), X=(\pi, 0,0)$, $M=(\pi, \pi, 0)$, and $Y=(0, \pi, 0)$ are the main symmetry points of the Brillouin zone.

Band structure calculations for manganites is more complicated than for cuprates for two reasons: a) the orbital ordering double the unit cell, b) the high spin values for $d^{4}(S=2)$ and for $d^{4 \pm 1}\left(S=5 / 2\right.$ for $d^{5}$ and $S=3 / 2$ for $\left.d^{3}\right)$ increase the number of states in the Hilbert space. The construction of the $X$-operators representation for $\mathrm{LaMnO}_{3}$ within the high spin $d^{3}, d^{4}$, and $d^{5}$ configurations has been done in Ref. 23 . For the undoped $\mathrm{LaMnO}_{3}$, the $\mathrm{LDA}+\mathrm{GTB}$ calculations result in the QP band structure with the large CT gap $E_{C T} \approx 2 \mathrm{eV}$. Above the top of the occupied valence band there is a virtual state with the activation energy $\Delta \varepsilon \approx 0.4$ $\mathrm{eV}$. Doping or non-stoichiometry transforms this state into the in-gap narrow band. The LDA+GTB calculations for hole-doped $\mathrm{La}_{1-x} \mathrm{Sr}_{x} \mathrm{MnO}_{3}$ for $x=0.2 \div 0.3$ resulted in the half-metallic ground state with the $100 \%$ spin polarization at the Fermi level in the ferromagnetic phase, that is illustrated in Fig. 5 .

Transition from the ferromagnetic to the paramagnetic phase results in the band narrowing $\propto \frac{1}{2} \cos (\theta)$ at $x=0$ that is two times stronger than the double exchange model provides 70 .

\section{Finite Temperature Effect on the Electronic Structure of $\mathrm{LaCoO}_{3}$}

The perovskite-oxide $\mathrm{LaCoO}_{3}$ has been studied intensely for many years due its unique magnetic properties and related insulator-metal transition (IMT) 71172 . A gradual appearance of the paramagnetism above $50 \mathrm{~K}$ from the diamagnetic ground state in called the spin-state transition. Goodenough was the first who 
suggested that instead of the Hund's rule dictated high spin (HS) $S=2$ the strong crystalline electric field results in the low spin (LS) $\mathrm{S}=0$ state for $d^{6}$ configuration of the $\mathrm{Co}^{3+}$ ion, and the energy difference is very small with the spin gap $\Delta_{S}=E(\mathrm{HS})-E(\mathrm{LS}) \sim 100 \mathrm{~K}$. The thermal population of the HS state provides the sharp increase of the magnetic susceptibility $\chi$ with a maximum around $100 \mathrm{~K}$. The nature of the excited spin state of $\mathrm{Co}^{3+}$ above the singlet ${ }^{1} A_{1 g}$ has been under debate (see for a recent review 73 ). Besides the original ${ }^{5} T_{2 g}$ HS state with the $t_{2 g}^{4} e_{g}^{2}$ configurations 72 there were many indications on the intermediate spin (IS) $S=1,{ }^{3} T_{1 g}$, state. The two stage model has been proposed with the LS-IS transition at $100 \mathrm{~K}$ and IS-HS transition at $550-600 \mathrm{~K}^{74 / 75}$. Recent electronic spin resonance $(\mathrm{ESR})$ 76, X-ray absorption spectroscopy (XAS) and X-ray magnetic circular dichroism (XMCD) 77 experiments prove that the lowest excited state is really the HS. Nevertheless the ${ }^{5} T_{2 g}$ term is splitted by the spin-orbital interaction in the low energy triplet with effective moment $J=1$, and higher energy sublevels with

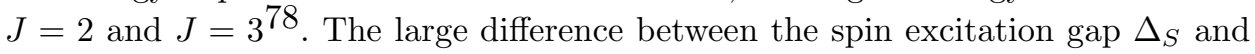
the charge gap given by the activation energy for electrical conductivity $E_{a} \approx 0.1 \mathrm{eV}$ at low $T$ indicates that $\mathrm{LaCoO}_{3}$ is not a simple band insulator 199 . The second shallow maximum in $\chi$ near $500 \div 600$ is often related to the insulator-metal transition. Surprisingly for the IMT electrical conductivity $\sigma$ does not seem to show any noticeable anomaly at this temperature 79 . Moreover the discrepancy between the large charge gap $2 E_{a} \approx 2300 \mathrm{~K}$ and the $T_{\mathrm{IMT}} \approx 600 \mathrm{~K}$ implies that the IMT cannot be simply argued in terms of a narrow-gap semiconductor 80 . We solved this problem by calculating the electronic band structure in the regime of strong electron correlations. We consider electron as the linear combination of quasiparticles (QP) given by excitations between the different multielectron configurations obtained by exact diagonalization of the $\mathrm{CoO}_{6}$ cluster. With the Hubbard operators constructed within the exact cluster eigenstates we can calculate the QP band structure for the infinite lattice. The QP spectral weight is determined by the occupation numbers of the local multielectron configurations. We find that the thermal population of different sublevels of the ${ }^{5} T_{2 g}$ HS term splitted by the spin-orbital interaction results both in the spinstate transition and also in some new QP excitations. Of particular importance is the hole creation QP from the initial $d^{6}$ HS into the $d^{5}$ HS term, this QP appears to form the in-gap state inside the large charge-transfer gap $E_{g} \approx 1.5 \mathrm{eV}$. The intercluster hopping transforms this local QP into the in-gap band that lies just under the bottom of empty conductivity band and provides the insulating gap $2 E_{a} \approx 0.2 \mathrm{eV}$ at $T=100 \mathrm{~K}$. It bandwidth increases with $T$, and overlapping with the conductivity band at $T=T_{\mathrm{IMT}}=587 \mathrm{~K}$ results in the IMT. Hence our approach allows to treat both the low $T$ spin-state transition and the high T IMT on the same footing.

$\mathrm{LaCoO}_{3}$ as well as other strongly correlated oxides is a difficult problem for the ab initio band theory. The LDA calculations 81 incorrectly predict a metal for paramagnetic $\mathrm{LaCoO}_{3}$. Various methods have been applied to study effect of 


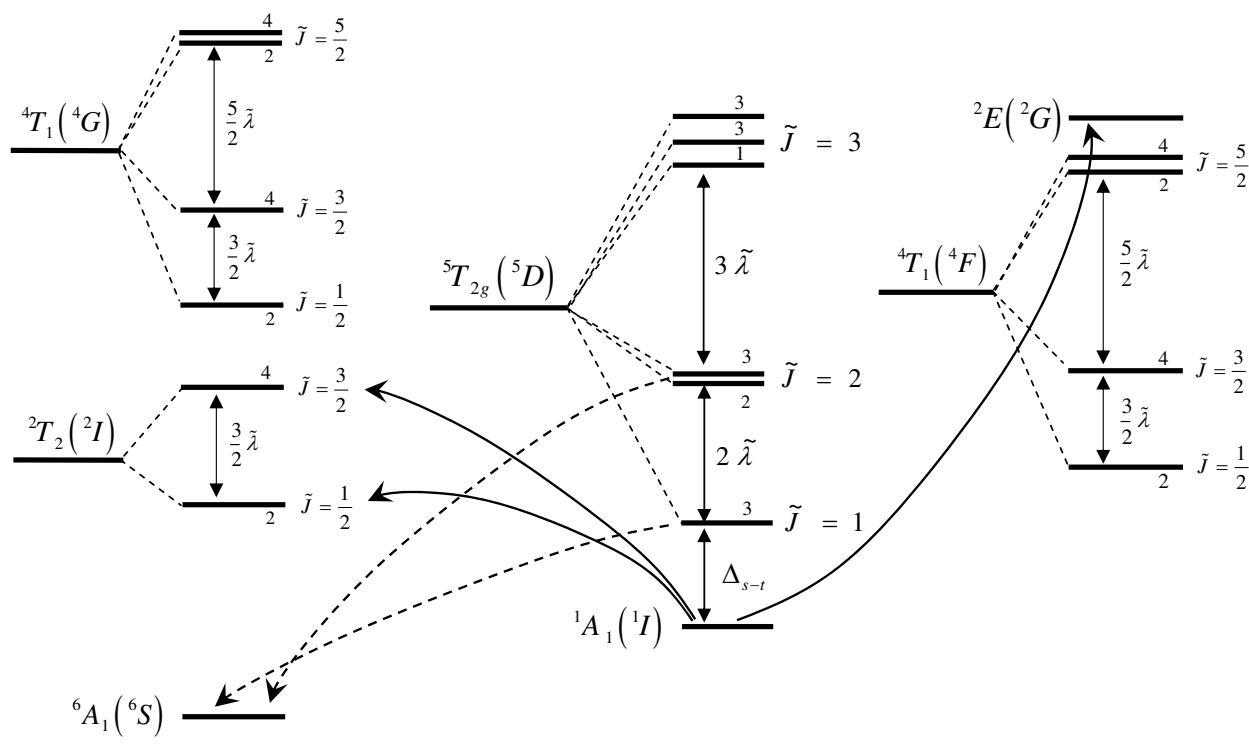
(a) $\mathrm{N}_{\mathrm{e}}=5$
(b) $\mathrm{N}_{\mathrm{e}}=6$
(c) $\mathrm{N}_{\mathrm{e}}=7$

Fig. 6. The low-energy part of the Hilbert space for $\mathrm{CoO}_{6}$ cluster with the electron numbers $N_{e}=5,6,7$. Terms with a given $N_{e}$ are the mixtures of $d^{N_{e}}, d^{N_{e}+1} \underline{L}$, and $d^{N_{e}+2} \underline{L}^{2}$ configurations. At $T=0$, only the $N_{e}=6$ low-spin term ${ }^{1} A_{1}$ is occupied; the Fermi-type excitations from this term which form the top of the valence band $\left(d^{6} \rightarrow d^{5}\right)$ and the bottom of the conductivity band $\left(d^{6} \rightarrow d^{7}\right)$ are shown by the solid lines with arrows. The dashed lines denote the in-gap excitations with the spectral weight increasing with temperature due to the population the HS excited $d^{6}$ terms.

correlations on the $\mathrm{LaCoO}_{3}$ electronic structure: $\mathrm{LDA}+\mathrm{U}$ or GGA $+\mathrm{U} 82|83| 84 \mid 85$, dynamical mean-field theory 86 . Recent variational cluster approximation (VCA) calculation 87 based on the exact diagonalization of the $\mathrm{CoO}_{6}$ cluster gives a reasonably accurate description of the low temperature properties: the insulating nature of the material, the photoelectron spectra, the LS-HS spin-state transition. The main deficiency of the VCA is the failure to reproduce the high temperature anomalies in the magnetic and electronic properties associated with the IMT.

The exact diagonalization of the multielectron Hamiltonian for a finite cluster provides a reliable general overview of the electronic structure of the correlated materials 88 . To incorporate the lattice effect several versions of the cluster perturbation theory are known $89[90$. To calculate the band dispersion in the strongly correlated material one has to go beyond the local multielectron language. The natural tool to solve this problem is given by the Hubbard $X$-operators $X_{f}^{p q}=|p\rangle\langle q|$ constructed with the $\mathrm{CoO}_{6}$ cluster eigenvectors $|p\rangle$ at site $\vec{R}_{f}$. All effects of the strong Coulomb interaction, spin-orbit coupling, covalence and the crystal field inside the $\mathrm{CoO}_{6}$ cluster are included in the set of the local eigenstates $E_{p}$. Here $p$ 

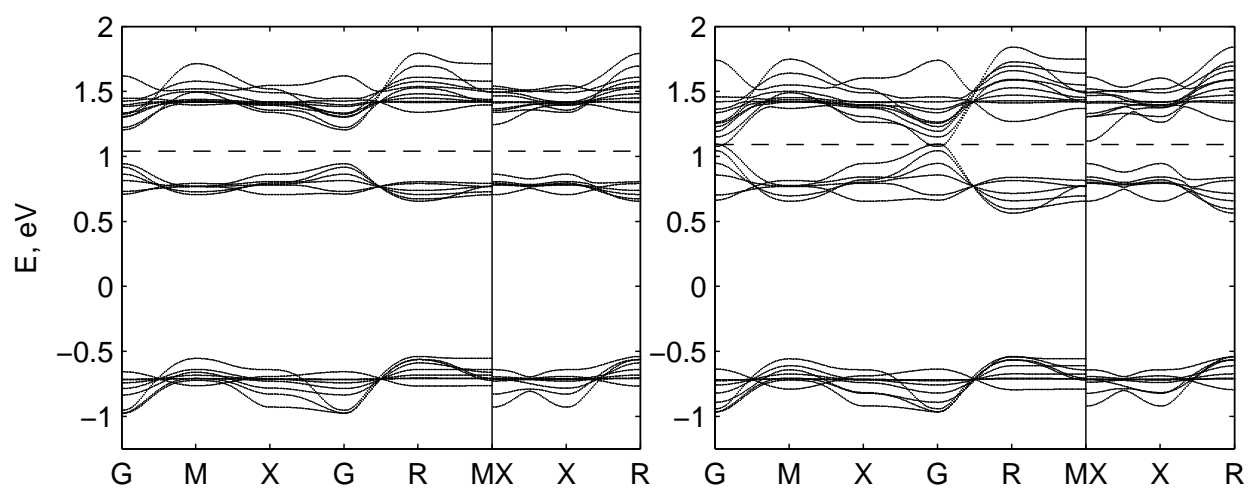

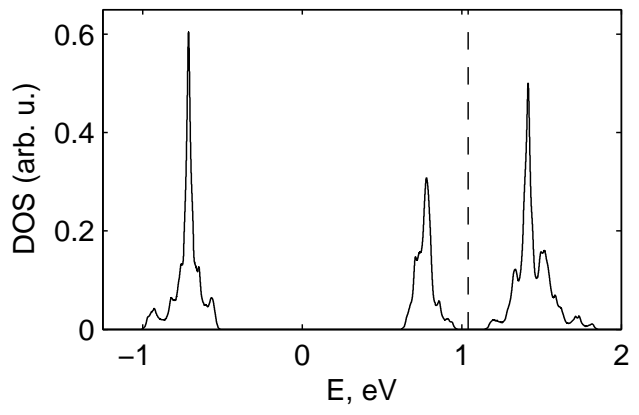

a

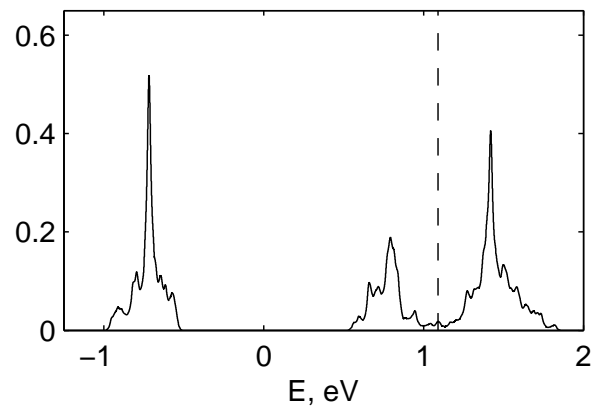

$\mathrm{b}$

Fig. 7. Quasiparticle dispersion and density of states at two different temperatures, $T=100 \mathrm{~K}$ (a) and $T=600 \mathrm{~K}(\mathrm{~b})$. At $T=0 \mathrm{~K}, \mathrm{LaCoO}_{3}$ is the charge-transfer insulator with the gap $E_{g} \approx 1.5 \mathrm{eV}$. At finite temperatures, the in-gap band appears below the conductivity band with the temperature dependent activation energy. At $T=100 \mathrm{~K}, E_{a} \approx 0.1 \mathrm{eV}$ (a). At $T=T_{\mathrm{IMT}}=587 \mathrm{~K}, E_{a}=0 \mathrm{eV}$, and above the $T_{\mathrm{IMT}}$ the band structure is of the metallic type (b).

denotes the following quantum numbers: the number of electrons (both $3 d$ Co and $p$ of O), spin $S$ and pseudoorbital moment $l$ (or the total pseudomoment $J$ due to spin-orbit coupling), the irreducible representation in the crystal field. A relevant number of electrons is determined from the electroneutrality, for stoichiometric $\mathrm{LaCoO}_{3}, n=6$. In the pure ionic model the corresponding energy level scheme for $d^{6} \mathrm{Co}^{3+}$ has been obtained in 78 . Due to the covalence there is admixture of the ligand hole configurations $d^{n+1} L$ and $d^{n+2} L^{2}$ that is very well known in the X-ray spectroscopy 91 . Contrary to spectroscopy the electronic structure calculations require the electron addition and removal excitations. For $\mathrm{LaCoO}_{3}$ it means the $d^{5}$ and $d^{7}$ configurations. The total low energy Hilbert space is shown in the Fig. 6. Here the energy level notations are the same as in the ionic mode $\frac{78}{\text { but all }}$ eigenstate contains the oxygen hole admixture due to the covalence effect. The calculation of the $n=5,6,7$ eigenvectors for $\mathrm{CoO}_{6}$ cluster with the spin-orbit coupling and the Coulomb interaction has been done in 92 .

The electron removal spectrum determines the top of the valence band, the 
corresponding electron quasiparticle QP are shown in the Fig. 6 by thin solid lines as the excitation from the ${ }^{1} A_{1} d^{6}$ singlet in the ${ }^{2} T_{2} d^{5}$ states with $J=1 / 2$ and $J=3 / 2$. There energies are

$$
\begin{aligned}
& \Omega_{V 1}=E\left(d^{6},{ }^{1} A_{1}\right)-E\left(d^{5},{ }^{2} T_{2}, \tilde{J}=1 / 2\right), \\
& \Omega_{V 2}=E\left(d^{6},{ }^{1} A_{1}\right)-E\left(d^{5},{ }^{2} T_{2}, \tilde{J}=3 / 2\right) .
\end{aligned}
$$

The bottom of empty conductivity band has the energy

$$
\Omega_{C}=E\left(d^{7},{ }^{2} E\right)-E\left(d^{6},{ }^{1} A_{1}\right) .
$$

All these bands have nonzero QP spectral weight. The intercluster hopping results in the dispersion, $\Omega_{n} \rightarrow \Omega_{n}(k)$. The QP LDA+GTB band structure corresponds to the insulator with the gap $E_{g} \approx 1.5 \mathrm{eV}$ (Fig. (7) at $T=0$. This gap value is rather close to the VCA gap $\frac{87}{1}$ and the experimental value $E_{g} \approx 1 \mathrm{eV}$.

At finite temperature, the thermal excitation over the spin-gap $\Delta_{S}$ into the $J=1$ and over the gap $\Delta_{S}+2 \lambda$ into the $J=2$ sublevels of the HS ${ }^{5} T_{2 g}$ state occurs. We take $\Delta_{S}=140 \mathrm{~K}$ and $\lambda=-185 \mathrm{~K}$ following Ref. 76. Partial occupation of the excited HS states results in the drastically change of the QP spectrum. For $T=0 \mathrm{~K}$, excitations from the ${ }^{1} A_{1} d^{6}$ singlet in the lowest ${ }^{6} A_{1} d^{5}$ term were forbidden due to spin conservation (the corresponding matrix element $\gamma_{n}=0$ ), and the excitation from $\left|d^{6}, \tilde{J}=1\right\rangle$ in $\left|d^{5},{ }^{6} A_{1}\right\rangle$ has nonzero matrix element (shown by dashed line $\Omega_{V 1}^{*}$ in Fig. (6) but zero filling factor as the excitation between two empty states. For $T \neq 0$, the filling factor for the $\Omega_{V 1}^{*}$ and $\Omega_{V 2}^{*}$ QP is nonzero and is equal to the occupation number $n_{1}$ and $n_{2}$ of the states $\left|d^{6}, \tilde{J}=1\right\rangle$ and $\left|d^{6}, \tilde{J}=2\right\rangle$ correspondingly. The energies of these QP are

$$
\begin{aligned}
& \Omega_{V 1}^{*}=E\left(d^{6},{ }^{5} T_{2 g}, \tilde{J}=1\right)-E\left(d^{5},{ }^{6} A_{1}\right), \\
& \Omega_{V 2}^{*}=E\left(d^{6},{ }^{5} T_{2 g}, \tilde{J}=2\right)-E\left(d^{5},{ }^{6} A_{1}\right) .
\end{aligned}
$$

Energies of these QP appear to be slightly below the bottom of the conductivity band, see DOS at finite temperature in Fig. 7 . Thus we have obtained that these temperature-induced QP states lies inside the charge-transfer gap, they are the in-gap states. Similar in-gap states are known to results from doping in the high temperature superconductors. The $\mathrm{LaCoO}_{3}$ is unique because the in-gap states are induced by heating. The chemical potential lies in the narrow gap $2 E_{a} \approx 0.2 \mathrm{eV}$ at $T=100 \mathrm{~K}$ between the in-gap states and conductivity band.

From the GTB dispersion equation (12) it is clear that the in-gap bandwidth is proportional to the occupation numbers $n_{1}$ and $n_{2}$ of the excited HS states. With further temperature increase, the in-gap bands $\Omega_{V 1}^{*}$ and $\Omega_{V 2}^{*}$ become wider and finally overlap with the conductivity band $\Omega_{C}$ (Fig. (7) at $T=T_{\mathrm{IMT}}=587 \mathrm{~K}$. It should be clarified that the IMT in $\mathrm{LaCoO}_{3}$ is not the thermodynamic phase 
transition, there is no any order parameter associated with the gap contrary to the classical IMT in $\mathrm{VO}_{2}$, NiS etc.

Thus, we find that a correct definition of the electron in strongly correlated system directly results in the in-gap states during the spin-state transition due to the thermal population of the excited HS states. Close to the spin-state temperature region the in-gap states determine the value of the activation energy $E_{a} \approx 0.1 \mathrm{eV}$. Further temperature increase results in large in-gap bandwidth and smaller $E_{a}$, and finally $E_{a}=0$ at $T_{\mathrm{IMT}}$. As concerns the weak maximum in the $\chi(T)$ close to the IMT, it may be a small Pauli-type contribution from the itinerant carriers above $T_{\mathrm{IMT}}$. We emphasize that instead of rather large difference in temperatures of the spin-state transition $(\sim 100 \mathrm{~K})$ and the IMT $(600 \mathrm{~K})$ the underlying mechanism is the same and is induced by the thermal population of the excited HS states.

\section{Conclusions}

We have presented the main ideas of the LDA+GTB method. Being invented to study the high- $T_{c}$ superconductivity in cuprates, LDA+GTB method appears to be powerful approach to systems with SEC and useful for other Mott insulators. Since it is a combination of the ab initio and model approaches, the method cannot go beyond the restriction of the model used in GTB. For example, the absence of the long-range Coulomb interaction which determines the Coulomb matrix elements in the large-wavelength limit prevents the correct description of the overdoped cuprates. Of course, it is the common deficiency of all Hubbard-type models. The modern version of the LDA+GTB cannot be used when the perturbation parameter $t / U$ increases and the Mott transition is expected. Nevertheless, it works in the most difficult for conventional band theory region of strong electronic correlations.

Application of the LDA+GTb method for cuprates revealed two critical points in the doping dependence. The first one is related to the change of the FS connectivity and logarithmic divergences of DOS and of electronic heat capacity parameter $\gamma$ at the optimal doping $p_{\text {opt }}=0.151$. The logarithmic enhancement of DOS leads to the maximum in the doping dependence of superconducting critical temperature $T_{c}$ at the same critical point $x=p_{\text {opt }}$. The second QPT is associated with the collapse of the electron-like FS pocket at $p \rightarrow p^{*}=0.246$ and results in the step singularities in DOS and in Sommerfeld parameter $\gamma$. We have found the depletion of the hole's kinetic energy below $p^{*}$ and ascribe it to the pseudogap formation at $p<p^{*}$. Thus the two energy scales in cuprates measured by $T_{c}$ and $T^{*}$ are both related to the QPTs and to the changes of the cuprate's electronic structure with doping. The underlying physics is tightly connected with the scattering on the spin fluctuations. While approach starting from the conventional Feynman diagrammatic expansion requires frequency-dependent self-energy to get the same results (see, e.g., CDMFT), $X$-operators technique allows to catch the crucial effects of scattering even on the mean-filed level.

The multiorbital extension of the same ideas results in a qualitatively correct 
description of the main peculiarities of the electronic properties of manganites and cobaltites. Further study of these and other Mott-Hubbard insulators are in progress.

\section{Acknowledgments}

We are thankful to O.K. Andersen, V.I. Anisimov, A.F. Barabanov, K.I. Kikoin, N.M. Plakida, S. Sakai, A.-M.S. Tremblay, V.V. Val'kov, and R.O. Zaitsev for useful discussions.

This work was supported by the Presisium of RAS program Quantum physics of condensed matter N 20.7, Grant "Leading scientific schools of Russia" (NSh 10442012.2), RFBR (Grant No. 09-02-00127), Integration Grant of SBRAS-UrBRAS N 44, Grant of President of Russia MK-1683.2010.2, FCP Scientific and Researchand-Educational Personnel of Innovative Russia for 2009-2013 (GK 16.740.12.0731 and GK 16.740.11.0740), and Siberian Federal University (Theme N F-11). M.M.K. and E.I.S. acknowledges support from The Dynasty Foundation and ICFPM.

\section{References}

1. P. Hohenberg and W. Kohn, Phys. Rev. 136, B864 (1964)

2. W. Kohn and L.J. Sham, Phys. Rev. 140, A1133 (1965)

3. R.O. Jones and O. Gunnarsson, Rev. Mod. Phys. 61, 689 (1989)

4. J.C. Hubbard, Proc. Roy. Soc. A 285, 542 (1965)

5. V.I. Anisimov, J. Zaanen, and O.K. Andersen, Phys. Rev. B 44, 943 (1991)

6. A. Svane and O. Gunnarsson, Phys. Rev. Lett. 65, 1148 (1990)

7. V.I. Anisimov, A.I. Poteryaev, M.A. Korotin, A.O. Anokhin, and G. Kotliar, J. Phys.: Condens. Matter 9, 7359 (1997)

8. A.I. Lichtenstein and M.I. Katsnelson, Phys. Rev. B 57, 6884 (1998)

9. K. Held, I.A. Nekrasov, N. Blümer, V.I. Anisimov, and D. Vollhardt, Int. J. Mod. Phys. B 15, $2611(2001)$

10. G. Kotliar, S.Y. Savrasov, K. Haule, V.S. Oudovenko, O. Parcollet, and C.A. Marianetti, Rev. Mod. Phys. 78, 865 (2006)

11. W. Metzner and D. Vollhardt, Phys. Rev. Lett. 62, 324 (1989)

12. D. Vollhardt, in Correlated Electron Systems, edited by V. J. Emery, World Scientific, Singapore, 1993, p. 57

13. A. Georges, G. Kotliar, W. Krauth, and M. Rozenberg, Rev. Mod. Phys. 68, 13 (1996)

14. M.H. Hettler, A.N. Tahvildar-Zadeh, M. Jarrell, T. Pruschke, and H.R. Krishnamurthy, Phys. Rev. B 58, R7475 (1998)

15. G. Kotliar, S.Y. Savrasov, G. Pálsson, and G. Biroli, Phys. Rev. Lett. 87, 186401 (2001)

16. M. Potthoff, Eur. Phys. J. B 32, 429 (2003)

17. T. Maier, M. Jarrell, T. Pruschke, and M.H. Hettler, Rev. Mod. Phys. 77, 1027 (2005)

18. S.Yu. Savrasov and G. Kotliar, Phys. Rev. B 69, 245101 (2004)

19. S.G. Ovchinnikov and I.S. Sandalov, Physica C 161, 607 (1989)

20. V.A. Gavrichkov, S.G. Ovchinnikov, A.A. Borisov, and E.G. Goryachev, JETP 91, 369 (2000)

21. Y.B. Gaididei, V.M. Loktev, Phys. Stat. Sol. B 147, 307 (1988)

22. M.M. Korshunov, V.A. Gavrichkov, S.G. Ovchinnikov, I.A. Nekrasov, Z.V. Pchelkina, V.I. Anisimov, Phys.Rev. B. 72, 165104 (2005) 
23. V.A. Gavrichkov, S.G. Ovchinnikov, L.E. Yakimov, JETP 102, 972 (2006)

24. S.G. Ovchinnikov, Yu.S. Orlov, I.A. Nekrasov, and Z.V. Pchelkina, arXiv:1005.1732

25. see Chapter. 5 in Theoretical Methods for strongly Correlated systems, Eds. A. Avella and F. Mancini (Springer Series in Solid-State Sciences 171, Springer, 2012), p. 505.

26. S.G. Ovchinnikov and V.V. Val'kov, Hubbard Operators in the Theory of Strongly Correlated Electrons. (Imperial College Press, London-Singapore, 2004), p. 241

27. D.N. Zubarev, Sov. Phys. Usp. 3, 320 (1960)

28. O.K. Andersen, Z. Pawlowska, O. Jepsen, Phys. Rev. B 34, 5253 (1986)

29. V.I. Anisimov, D.E. Kondakov, A.V. Kozhevnikov, I.A. Nekrasov, Z.V. Pchelkina, J.W. Allen, S.-K. Mo, H.-D. Kim, P. Metcalf, S. Suga, A. Sekiyama, G. Keller, I. Leonov, X. Ren, and D. Vollhardt, Phys. Rev. B 71, 125119 (2005)

30. O.K. Andersen, T. Saha-Dasgupta, Phys. Rev. B 62, 16219 (R) (2000)

31. V.A. Gavrichkov, S.G. Ovchinnikov, I.A. Nekrasov, E.E. Kohorina, Z.V. Pchelkina, Physics of the Solid State 49, 2052 (2007)

32. O. Gunnarsson, O.K. Andersen, O. Jepsen, and J. Zaanen, Phys. Rev. B 39, 1708 (1989)

33. V.I. Anisimov, M.A. Korotin, I.A. Nekrasov, Z.V. Pchelkina, and S. Sorella, Phys. Rev. B 66, 100502(R) (2002)

34. R. Raimondi and J.H. Jefferson, L.F. Feiner, Phys. Rev. B 53, 8774 (1996)

35. J. Zaanen, G.A. Sawatzky, J. Solid State Chem. 88, 8 (1990)

36. R.O. Zaitsev, JETP 43, 574 (1976)

37. J. Zaanen, G.A. Sawatzky, J.W. Allen, Phys. Rev. Lett. 55, 418 (1985)

38. B. Westwanski, A. Pawlikovski, Phys. Lett. A 43, 201 (1973)

39. V.G. Bar'yakhtar, V.N. Krivoruchko, and D.A. Yablonskii, Green's Functions in Magnetism Theory, in Russian. (Nauk. Dumka, Kiev, 1984), p. 336

40. S.G. Ovchinnikov, Phys. Rev. B 49, 9891 (1994)

41. N.M. Plakida and V.S. Oudovenko, JETP 104, 230 (2007)

42. N. Harrison, R.D. McDonald, and J. Singleton, Phys. Rev. Lett. 99, 206406 (2007)

43. A.F. Barabanov, R. Hayn, A.A. Kovalev, O.V. Urazaev, and A.M. Belemuk, JETP 92, 677 (2001)

44. M.M. Korshunov and S.G. Ovchinnikov, Eur. Phys. J. B 57, 271 (2007)

45. P. Prelovšek, Z. Phys. B 103, 363 (1997)

46. N.M. Plakida and V.S. Oudovenko, Phys. Rev. B 59, 11949 (1999)

47. Z. Liu and E. Manousakis, Phys. Rev. B 45, 2425 (1992)

48. H. Shimahara and S. Takada, J. Phys. Soc. Jpn. 60, 2394 (1991)

49. V. Val'kov and D.M. Dzebisashvili, JETP 100, 608 (2005)

50. L. Hozoi, M.S. Laad, and P. Fulde, Phys. Rev. B 78, 165107 (2008)

51. S. Chakravarty and H.-Y. Kee, Proc. Nat. Acad. Sc. (USA) 105, 8835 (2008)

52. S. Sachdev, A.V. Chubukov, and A. Sokol, Phys. Rev. B 51, 14874 (1995)

53. I.M. Lifshitz, M.Y. Azbel, and M.I. Kaganov 1972 Electron theory of Metals (Consultant Press, New York); I.M. Lifshitz, Zh. Eksp. Teor. Fiz. 38, 1569 (1960) [Sov. Phys. JETP 11, 1130 (1960)]

54. J.M. Ziman 1964 Principles of the theory of solids (Cambridge University Press, Cambridge)

55. S.S. Nedorezov, Zh. Eksp. Teor. Fiz. 51, 868 (1966) [Sov. Phys. JETP 24, 578 (1967)]

56. K. Mikelsons, E. Khatami, D. Galanakis, A. Macridin, J. Moreno, and M. Jarrell, Phys. Rev. B 80, 140505(R) (2009)

57. E.I. Shneyder and S.G. Ovchinnikov, JETP Lett. 83, 394 (2006)

58. J.R. Cooper and J.W. Loram, J. Phys. IV France 10, Pr3-213 (2000)

59. J.W. Loram, J. Luo, J.R. Cooper, W.Y. Liang, and J.L. Tallon, J. Phys. Chem. Solids 
62, 59 (2001)

60. A. Georges, G. Kotliar, W. Krauth, and M. Rozenberg, Rev. Mod. Phys. 68, 13 (1996)

61. D. Sénéchal, P.-L. Lavertu, M.-A. Marois, and A.-M.S. Tremblay, Phys. Rev. Lett. 94, $156404(2005)$

62. S.S. Kancharla, B. Kyung, D. Sénéchal, M. Civelli, M. Capone, G. Kotliar, and A.M.S. Tremblay, Phys. Rev. B 77, 184516 (2008)

63. K. Haule and G. Kotliar, Phys. Rev. B 76, 104509 (2007)

64. A. Macridin, M. Jarrell, T. Maier, and G.A. Sawatzky, Phys. Rev. B 71, 134527 (2005)

65. B. Kyung, D. Sénéchal, and A.-M.S. Tremblay, Phys. Rev. B 80, 205109 (2009)

66. S. Sakai, Y. Motome, and M. Imada, Phys. Rev. Lett. 102, 056404 (2009)

67. S. Sakai, Y. Motome, and M. Imada, Phys. Rev. B 82, 134505 (2010)

68. S.G. Ovchinnikov, V.N. Zabluda, JETP 98, 135 (2004)

69. S.G. Ovchinnikov, B.A. Gizhevskii, N.V. Kazak, V.V. Rudenko, A.V. Telegin, JETP Lett. 90, 519 (2009)

70. V.A. Gavrichkov, S.G. Ovchinnikov, Z.V. Pchelkina, J. Phys.: Conference Series 200, 012046 (2010)

71. G.H. Jonker and J.H. Van Santen, Physica 19, 120 (1953).

72. P.M. Raccah and J.B. Goodenough, Phys. Rev. 155, 932 (1967).

73. N.B. Ivanova, S.G. Ovchinnikov, M.M. Korshunov, I.M. Eremin and N.V. Kazak, Physics-Uspekhi 52, 789 (2009).

74. R.H. Potze, G.A. Sawatzky, and M. Abbate, Phys. Rev. B 51, 11501 (1995).

75. T. Saitoh, T. Mizokawa, A. Fujimori, M. Abbate, Y. Takeda, and M. Takano, Phys. Rev. B 55, 4257 (1997).

76. S. Noguchi, S. Kawamata, K. Okuda, H. Najiri, and M. Motokawa, Phys. Rev. B 66, 094404 (2002).

77. M.W. Haverkort, Z. Hu, J.C. Cezar, T. Burnus, H. Hartmann, M. Reuther, C. Zobel, T. Lorenz, A. Tanaka, N.B. Brookes, H.H. Hsieh, H.-J. Lin, C.T. Chen, and L.H. Tjeng, Phys. Rev. Lett. 97, 176405 (2006).

78. Z. Ropka and R.J. Radwanski, Phys. Rev. B 67, 172401 (2003).

79. S. Yamaguchi, Y. Okimoto, H. H. Taniguchi, and Y. Tokura, Phys. Rev. B 53, R2926 (1996).

80. S. Yamaguchi, Y. Okimoto, and Y. Tokura, Phys. Rev. B 54, R11022 (1996).

81. P. Ravindran, P.A. Korzhavye, H. Fjellvag, and A. Kjekshus, Phys. Rev. B 60, 16423 (1999)

82. M.A. Korotin, S.Yu. Ezhov, I.V. Solovyev, and V.I. Anisimov, Phys. Rev. B 54, 5309 (1996).

83. K. Knizek, P. Novak, and Z. Jirak, Phys. Rev. B 71, 054420 (2005).

84. S.K. Pandey, A. Kumar, S. Patil, V.R.R. Medicherla, R.S. Singh, K. Maiti, D. Prabhakaran, A.T. Boothroyd, and A.V. Pimpale, Phys. Rev. B 77, 045123 (2008).

85. H. Hsu, K. Umemoto, M. Coccoccioni, and R. Wentzcovitch, Phys. Rev. B 79, 125124 (2009).

86. L. Craco and E. Muller-Hartmann, Phys. Rev. B 77, 045130 (2008).

87. R. Eder, Phys. Rev. B 81, 035101 (2010).

88. E. Dagotto, Rev. Mod. Phys. 66, 763 (1994).

89. T. Maier, M. Jarrel, Th. Pruschke, and M.H. Hettler, Rev. Mod. Phys. 77, 1027 (2005).

90. D. Senechal and A.-M. Tremblay, Phys. Rev. Lett. 92, 126401 (2004).

91. M. Abbate, J.C. Fuggle, A. Fujimori, L.H. Tjeng, C.T. Chen, R. Potze, G.A. Sawatzky, H. Eisaki, and S. Uchida, Phys. Rev. B 47, 16124 (1993).

92. Yu.S. Orlov and S.G. Ovchinnikov, JETP 109, 322 (2009). 